\title{
Synobins: An Intermediate Level towards Annotation and Semantic Retrieval
}

\author{
Daniela Stan Raicu ${ }^{1}$ and Ishwar K. Sethi ${ }^{2}$ \\ ${ }^{1}$ Intelligent Multimedia Processing Laboratory, School of Computer Science, Telecommunications, and Information Systems, \\ DePaul University, Chicago, IL 60604, USA \\ ${ }^{2}$ IIE Laboratory, Department of Computer Science \& Engineering, Oakland University, Rochester, MI 48309-4478, USA
}

Received 1 September 2004; Revised 24 March 2005; Accepted 9 May 2005

\begin{abstract}
To reason about the meaning of an image, useful information should be provided with that image; however, images often contain little to no textual information about the objects they are depicting, which is the precise reason why there is a need for CBIR systems that exploit only the correlations present in the raw pixel data. In this paper, we proposed a new type of image feature, which consists of patterns of colors and intensities that capture the latent associations among images and primitive features in such a way that the noise and redundancy are eliminated. We introduced the synobin, a new term for content-based image retrieval literature, which is the equivalent of a synonym word from text retrieval, to name the bin that is synonymous with other bins of a color feature, in the sense that they are similarly used across the image database. In a formal definition, a group of synobins is given by the most important bins participating in forming of a useful pattern, that is, the bins having the highest coefficients in the linear combination defining that pattern. Incorporating our feature model into a CBIR system moves the research in image retrieval beyond simple matching of images based on their primitive features and creates a ground for learning image semantics from visual content. A system developed using our proposed feature model will have the capability of learning associations not only between semantic concepts and images, but also between semantic concepts and patterns. We evaluated the performance of our system based on the retrieval accuracy and on the perceptual similarity order among retrieved images. When compared to standard image retrieval methods, our preliminary results show that even if the feature space was reduced to only $3 \%-5 \%$ of the initial space, the accuracy and perceptual similarity for our system remain the same or better depending on the category of images.
\end{abstract}

Copyright $\odot 2006$ Hindawi Publishing Corporation. All rights reserved.

\section{INTRODUCTION}

In the last ten years, the multimedia superhighway has expanded exponentially, bringing vast repository of information to the desktop in a few mouse clicks; therefore, there is an evergrowing demand for tools to locate information by content with greater accuracy and efficiency. In particular, methods for content-based image retrieval (CBIR) have drawn the most attention as many of the underlying techniques can be easily applied to other multimedia artifacts with some suitable modifications. A CBIR system can be viewed as two main components: feature extraction and the search for similar images in a feature space.

The first component is meant to provide useful information in the form of features about the meaning or the content of an image. Features may be extracted either from text coming from different sources (captions, transcripts, text surrounding images on Web, etc.) or directly from raw image data (arrays of pixel intensities). Within this component, there are several concerns that might prevail. When images contain no textual description, there is a need for systems that exploit only the later features, well known as visual or low-level features (color, texture, and shape). Furthermore, the meaning of an image should be derived from the whole structure of the image and not from individual parts [1]. For instance, if the color information is used to extract the features, an individual color histogram bin carries little information by itself, but in combination with some other similar bins can contribute to the mood of the image (such as harmony, happiness) and is a useful clue for the image content description. Finally, the meaning of an image should be derived in some context. Let us assume that an image database is organized in a way that preserves the relationships between images with respect to a certain similarity metric. Similar images are grouped together and the formed clusters can be considered different contexts of the image database. The meaning of an image will be derived from the interpretations of the images of the cluster in which the image appears.

The second component deals with the process of searching for similar images to a given query. Searching for 
content-based similarity involves two main issues: defining similarity metrics, and defining data structures to facilitate fast search and retrieval. When the image features correspond to low-level features, simple similarity metrics such as normalized Euclidean distance may not simulate the human perception of a certain visual concept. Metrics that are more complicated need to be defined to produce similarity results closer to human perception. The complexity of the similarity measures also increases with the high dimensionality of the feature space; furthermore, standard data structure indexing techniques perform poor for dimensions exceeding ten [2]. A possible solution towards solving these problems is to apply a dimension reduction stage before defining a data indexing structure that supports non-Euclidean similarity metrics.

The approach that we are presenting in this paper falls within the first component of a CBIR system. Using latent semantic indexing (LSI) technique from information retrieval, we propose a new type of image feature that is meant to capture the hidden associations among visual feature elements within an image and across the image database. We implement and describe our system using only color information, but any other feature (visual or text-based) can be easily incorporated in our system since the information contained in LSI is given by the notion of occurrence, and not by numeric abstract values [3]. The new image features, named patterns, are defined as linear combinations of feature elements, in which the combinations' coefficients weight the elements' importance in forming the corresponding new features. Following our approach, the meaning or the content of an image will not require recognition of objects present in the image but rather identification of patterns of color and intensities in the image. Moreover, the property of LSI to discover hidden associations among images gives our system the capability to create contexts (groups of similar images) in which the meaning of an image would be interpreted. As a result, instead of trying to give all possible meanings to an image, the system interprets an image based on the discovered patterns and in the context in which the image appears.

Our system also brings a major contribution to the second component of a CBIR system. It is a promising approach in solving the high-dimensionality problem since combinations modeling only the real patterns and not the peculiarities in the database are considered as new features. It provides a new type of retrieval, specifically color retrieval in the context: an image, even if it is not indexed by the query feature elements, could be retrieved because it shares the same color patterns with another image that is relevant to the query image.

\section{RELATED WORK AND OUR CONTRIBUTION}

Visual feature extraction in the context of content-based image retrieval has been studied extensively in the last years. Most of the work concentrates on low-level visual features (such as color, shape, texture) and adopts a feature-based image retrieval approach. Examples include the QBIC system [4], the Virage system [5], the RetrievalWare system [6], the VisualSEEk and WebSEEK systems [7], the Mars system [8], and Netra [9].
QBIC (query by image content) was developed by IBM in 1994 and since then the system has gone through continuous development. Currently, it supports queries by examples based on several features such as color, texture, and user-constructed sketches. The QBIC version for stock photos provides additional options for image features such as color histogram, layout, texture, and special hybrid color. VIR (visual information retrieval) image engine was developed by Virage, Inc., in 1996; the system uses the concept "primitives" to express a given feature's representation and incorporates, in addition to the general features (color, texture, and shape), domain-specific features tailored for different applications (such as satellite imaging). Visual RetrievalWare was developed by Excalibur Technologies Corp. and it provides similarity measures for individual or combinations of low-level general features (color content, shape content, texture content, brightness structure, color structure, and aspect ratio). The techniques of the system are currently incorporated in the Image Surfer option of Yahoo search engine.

In the academic world, several CBIR systems have also been developed. VisualSEEk and WebSEEK systems were designed at Columbia University and they allow Web queries using keywords and visual features. One system that has been a truly interdisciplinary effort is MARS (multimedia analysis and retrieval system) developed at the University of Illinois at Urbana-Champaign. It attempts to integrate computer and human qualities, using both the CBIR technology and the user feedback at a number of levels in order to make the retrieval more relevant to the end user. NETRA is a prototype system developed in the Alexandria Digital Library (ADL) Project at the University of California at Santa Barbara, USA. The Netra system uses color texture, shape, and spatial location information to provide region-based searching based on local image properties instead of just image-based retrieval.

There are many other CBIR systems that focus on different research issues with respect to the visual image features and the interested reader may refer to the CBIR report by Eakins and Graham [10]. However, the application of these systems to real-world problems is limited because of the lack of semantic meanings in extracted features; specific low-level image features may provide a solution to image retrieval in some applications, but may have a problem in handling other applications. If at some point, standards can be developed and agreed upon, an integration of visual and textual thesauri or extraction of meaningful visual features may well be the answer for many image collections in the future. Our approach presented in this paper proposes a new type of color features (color patterns) and acts as an intermediate step towards meaningful retrieval. Since the technique of our approach is based on latent semantic indexing, we will focus our discussion on the use of this technique for image retrieval.

Latent semantic indexing (LSI) for text retrieval was introduced by Dumais et al in 1990. Its capability of inducing and representing meanings of words and documents reflected in their usage made the technique popular and a powerful tool for automatic text indexing and retrieval. It provides retrieval based on the conceptual content of 
a document instead of term-matching retrieval. This is very important since users want to retrieve based on conceptual content and individual terms provide unreliable evidence about the conceptual topic or meaning of a document. Several attempts were made to exploit the benefits of LSI in the content-based image retrieval (CBIR) field.

In 1998, La Cascia et al. [11] proposed the LSI technique to extract semantic content in the form of keywords from text surrounding the images on the web (alt-tags, title, near text). Then, the LSI textual representation for an HTML document was associated with each visual representation of the images contained therein. The visual representation was derived using color histogram and dominant orientation histogram, and principal component analysis (PCA) technique was used to reduce the dimension of the visual features. Their experimental results show that the retrieval accuracy increases when both visual and textual information are used. In 1999, Westerveld et al. [12] proposed an integrated framework, in which first the terms and the visual content are combined and then LSI is applied on the unified vector to capture the associations between images and text. The difference between PCA and singular value decomposition (the underlying technique on which LSI is based) is that PCA is applied on multiple observations while SVD is applicable on single observations [13].

The two approaches assume that some textual information is provided with the images. However, sometimes there is no textual information available for an image database or is very little (such as for photo galleries on Web). Therefore, there is a need for content-based retrieval systems that exploit the correlations present in the visual content of images, the pixel data. The first attempt to establish a general framework, keyblock, capable of capturing the correlations among image blocks by using text retrieval techniques was made by Rao et al. in $[14,15]$. In their framework, they propose $N$ blocks models which are $N$-gram models in the language modeling to extract comprehensive image features. The representative blocks, regions, or objects of an image form the keyblocks, the analog of keywords from text retrieval, and the correlations among these keyblocks are explored using the $N$-blocks models.

In our approach, no segmentation or blocks generation is necessary since we are taking into account only the correlations among the pixel values (unigram approach) and not their spatial and neighborhood properties. Furthermore, we propose a new type of image feature that will allow retrieval and semiautomatic across-image annotation based on groups of image data rather on mere pixels. The idea of grouping image data for semiautomatic across-image labeling was also used by Minka and Picard in [16]. They used a clustering approach to form within-image groups of image regions; these groups were used either directly for segmentation or for the computation of across-image groupings used in annotation. However, since clustering deals with only one type of entity (feature or image), the clustering approach had to be applied one more time on the within-image groups in order to derive the across-image groupings. Our approach makes use of LSI capability of dealing with different types of entities at the same time, and thus both low-level feature elements and images are considered together as the input. The output is a new feature space in which features and images are expressed as points, and the coordinates of each point derive the importance of the corresponding feature element in forming the new patterns. The highest values of the coordinates represent the most important bins participating in forming a useful pattern and they are called synobins. If there are any groups of feature element points or image points in this space, they will produce within or across contexts for the image database, respectively. Therefore, in addition to the dimension reduction property of LSI previously used by Niblack et al. in [17] to achieve scalable image retrieval, we exploit the LSI property of creating clusters of co-occurring features having the goal of generating image patterns, the analog of concepts derived from co-occurring terms in text retrieval. Figure 1 shows an example of a cluster of color bins and images obtained applying LSI on the color histograms used as images' visual content representations.

Since the images in the cluster have the semantic meaning, the textual characterization "sunset" can also be associated with the color pattern modeled by the cluster's bins. This association shows how the proposed approach can be used as an important step towards transforming the low-level features to meaningful high-level features.

As a result, instead of looking at an image as a meaningless pixel matrix and at a database as a sequence of images that make no sense when placed near each other, we create contexts in which meaningful perceptual impressions can be formed about the content and the similarity among images. In our approach, the within-image groupings will not serve for segmentation, but rather for recognition of patterns of color and intensities in the image.

Figure 2 shows the placement of LSI method as a component of a content-based image retrieval system. We briefly described the color pattern extraction in our previous paper [18] and we will emphasize the applicability of the color patterns to image retrieval and annotation in this paper.

\section{IMAGE LATENT SEMANTIC INDEXING MODEL}

The use of LSI for text retrieval involves the transformation of the document database into a matrix of terms by documents. Such a matrix is obtained by first doing preprocessing of the documents to filter out the stop words and converting the remaining words to their root form through stemming; then each term-vector representation of a document represents a column in the term-document matrix. An entry in this matrix is the frequency of a given word in a given document.

\subsection{The analogy between textual terms and image color bins}

To integrate the LSI approach in the field of image retrieval, first we need to define what kind of "terms" are images composed of. While in text databases, the raw material (words stored as ASCII character strings) has already been logically 


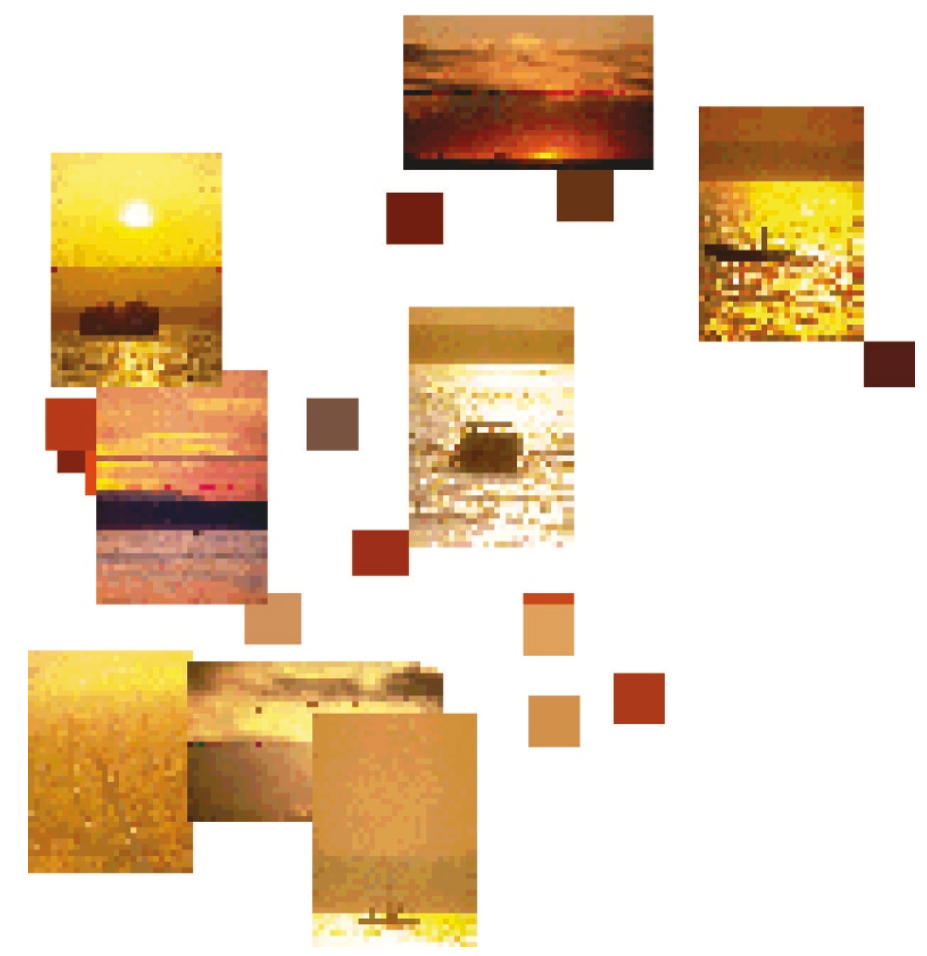

FIGURE 1: Example of a region in the LSI space used for image content interpretation. The small rectangles represent the color bins modeling the real color patterns present in the images from the corresponding region/cluster. The bins depicted produce a within context while the images produce an across context.

structured by Santini and Jain [1], in image databases, the raw material (arrays of pixel intensities) is unstructured and does not have any inherent meaning (Figure 3 ). To reason about the meaning or the content of an image, useful information has to be provided with that image. However, images often contain no textual description about the objects that they are depicting. Therefore, there is a need to extract useful information, in the form of low-level features, directly from the raw image data.

The issue of choosing the features to be extracted should be guided by the following concerns. First, the features should carry enough information about the image and should not require any domain-specific knowledge for their extraction. Second, the features should be easy to compute for the approach to be feasible for a large image collection and rapid retrieval. Finally, the features should relate well with the human perceptual characteristics since users will finally determine the suitability of the retrieved images. Because of perception subjectivity, there are multiple representations characterizing a given feature from a different perspective.

We describe our system using global histograms to represent the color information. The advantages of using global histograms are their robustness to translation and rotation about the viewing axis, slow modification with change in viewpoint and scale, and occlusion [19]. To capture the human perception into the representation, we choose the HSV space to represent the color information; this space correlates well with the human perception and is commonly used by artists to represent color information present in images [20]. After the color space transformation, three histograms (hue, saturation, and value) are calculated, and thus every image is encoded by an $m$-dimensional feature vector $(m=3 \times M$, 3 represents the number of histograms and $M$ stands for the number of bins of every histogram). At the end of this process, every image has a structure in the form of a color histogram and the histogram bins would be the equivalent of the "terms" of a document obtained after filtering out the stop words and stemming. As in the case of documents, an analysis of the importance of the bins within a feature vector representation shows that not all bins are equal for describing image content. To capture this fact, an information-theoretic weighting step can be applied to measure the overall importance of bins in the image database (intersimilarity) and the importance of bins within a particular image (intraimage characterization). Our bin-weighting scheme is the equivalent of the most effective term-weighting scheme from information retrieval, which is the term frequency-inverse document frequency (TF-IDF) scheme [21].

It is worth to mention here that LSI extracts the latent relationships among different terms from their relative usage without considering their order or spatial information. This motivated us to define the image "terms" with respect to a global histogram representation instead of a local one. 


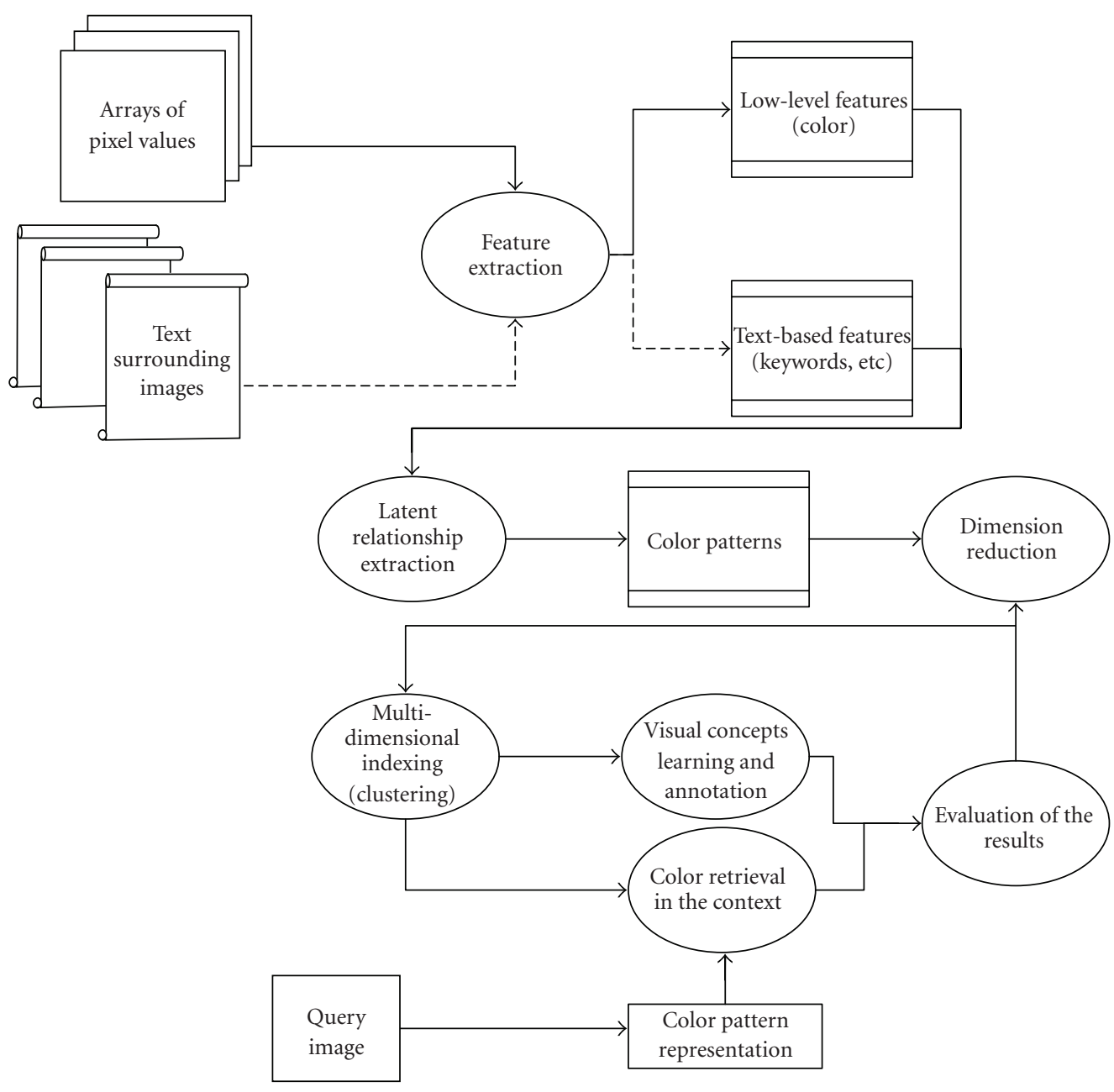

Figure 2: The diagram of the suggested system. The dashed lines represent unimplemented features that could be incorporated into the system.

However, a global histogram loses the spatial information, and thus the discrimination power of the histogram is saturated in the context of very large image databases. To keep up performance, additional low-level features (such as texture and shape) can be incorporated to bring more information about images from the database. Since latent semantic indexing is based on frequencies and not on actual values, before concatenating their vector value representations, the texture, and shape features will be quantized or clustered into discrete bins (tokens in information retrieval). Then, the obtained bins for texture and shape will be concatenated with the bins for color, and the LSI technique will be applied on the concatenated vector representing the three types of features. The explosion of dimensions of the histogram, produced by the incorporation of additional features, will be overcome by the LSI property of considering the quality of the information along each dimension; LSI reduces the highdimensional low-level feature space such that noise and redundancy are eliminated. Furthermore, the LSI technique will find the latent associations between the color bins, tex- ture bins, and shape bins, and the semantic meanings of the images present in the database.

\subsection{Pattern extraction}

\section{Singular value decomposition}

After the preprocessing stage, the image database is transformed into a matrix $W_{0}$, which is composed of bins (rows of $W_{0}$ ) by images (columns of $W_{0}$ ), and each entry represents the weight of a given bin in a given image:

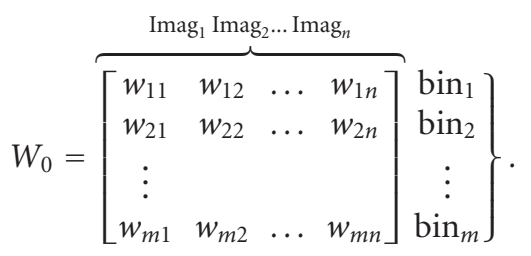

Singular value decomposition (SVD), the statistical method on which LSI is based, is performed on this matrix. By 


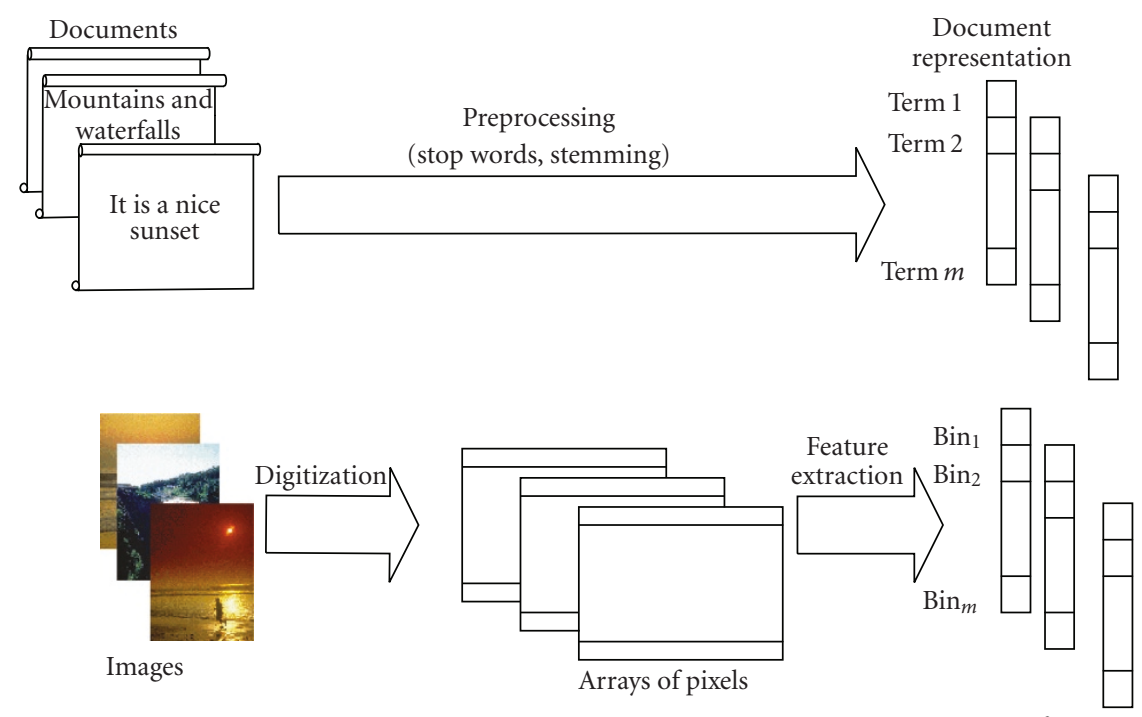

Image feature

representation

Figure 3: Document and image vector representation.
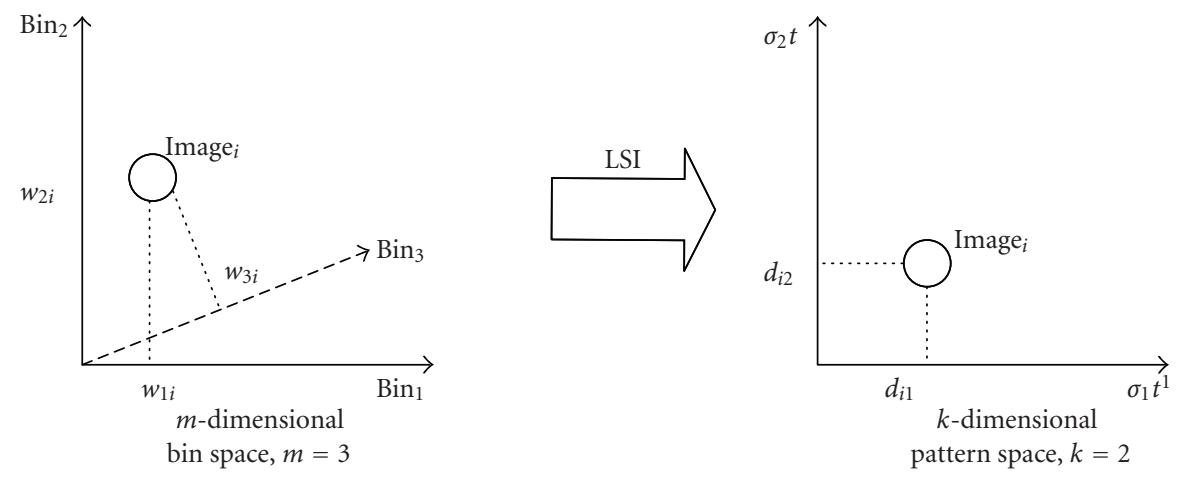

FIgURE 4: Mapping from a 3-dimensional low-level feature space to a 2-dimensional pattern space produced by LSI.

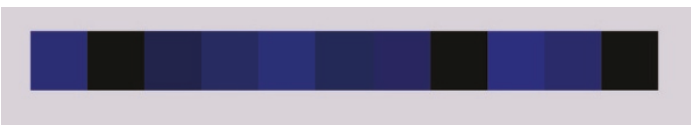

FIGURE 5: The first eleven color bins of a color pattern obtained $_{j}$ using LSI approach. We choose to illustrate the pattern with the most important bin as the leftmost one and such that the importance decreases as you go from left to right; as described previously, the importance is given by the elements of the vector $t^{j}$.

definition [22], the SVD of $W_{0}$ is any factorization of the form

$$
W_{0}=T_{0} \times \sum_{0} \times D_{0}^{\prime}
$$

where $T_{0}, D_{0}$ are two $m \times m$ ( $m=$ number of bins) and $n \times n$ ( $n=$ number of images) orthonormal matrices, respectively, and $\sum_{0}$ is an $m \times n$ diagonal matrix,

$$
\sum_{0}=\operatorname{diag}\left(\sigma_{1}, \sigma_{2}, \ldots, \sigma_{p}\right), \quad p=\min (m, n),
$$

such as the diagonal elements have the property that $\sigma_{1} \geq$ $\sigma_{2} \geq \cdots \geq \sigma_{p} \geq 0$ and they are the singular values of $W_{0}$. The main idea behind the SVD technique is that proper choice of $T_{0}$ and $D_{0}$ makes most of $\sigma_{i}$ zero; that is, most of the important information gets concentrated in a few dimensions. Let $k, k \leq p$, be the number of the first dimensions that contain this information; the remaining smaller singular values are set to zero. Since zeros are introduced into $\sum_{0}$, the representation can be simplified by deleting the rows and columns of $\sum_{0}$ to obtain a reduced $k \times k$ diagonal matrix $\sum$; then, deleting the corresponding columns of $T_{0}$ and $D_{0}^{\prime}$, two reduced matrices $T$ and $D^{\prime}$ of dimension $m \times k$ matrix and $k \times n$ matrix, respectively, are obtained. This result in a reduced model

$$
W=T \times \sum \times D^{\prime}
$$




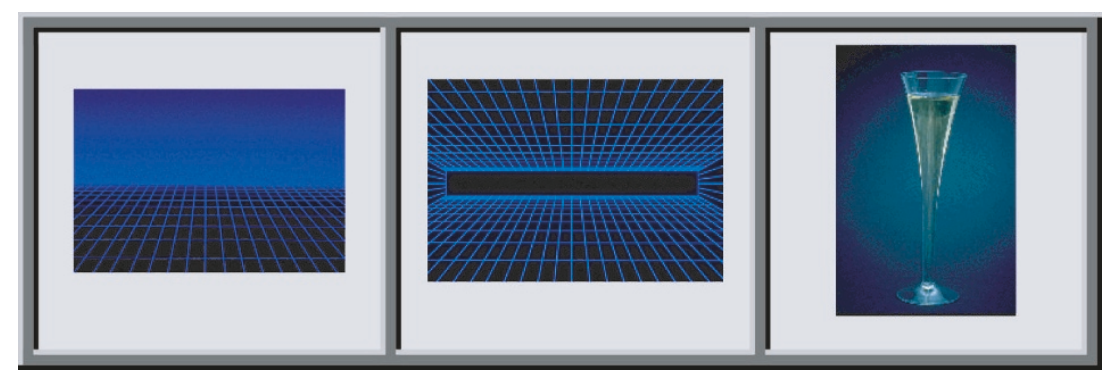

FIGURE 6: The first three images in which the color pattern from Figure 5 is mostly present; these images are given by the three highest values of the vector $d_{j}$ corresponding to the chosen pattern.

\begin{tabular}{|l|c|c|}
\hline & Text domain & Image domain \\
\hline $\begin{array}{c}\text { Same root/color, but } \\
\text { used in different contexts }\end{array}$ & Doctor, doctorate & \\
\hline $\begin{array}{c}\text { Different root/color, but } \\
\text { synonym/synobin }\end{array}$ & Doctor, physician & \\
\hline
\end{tabular}

Figure 7: Summary of the analogy between synonyms and synobins.

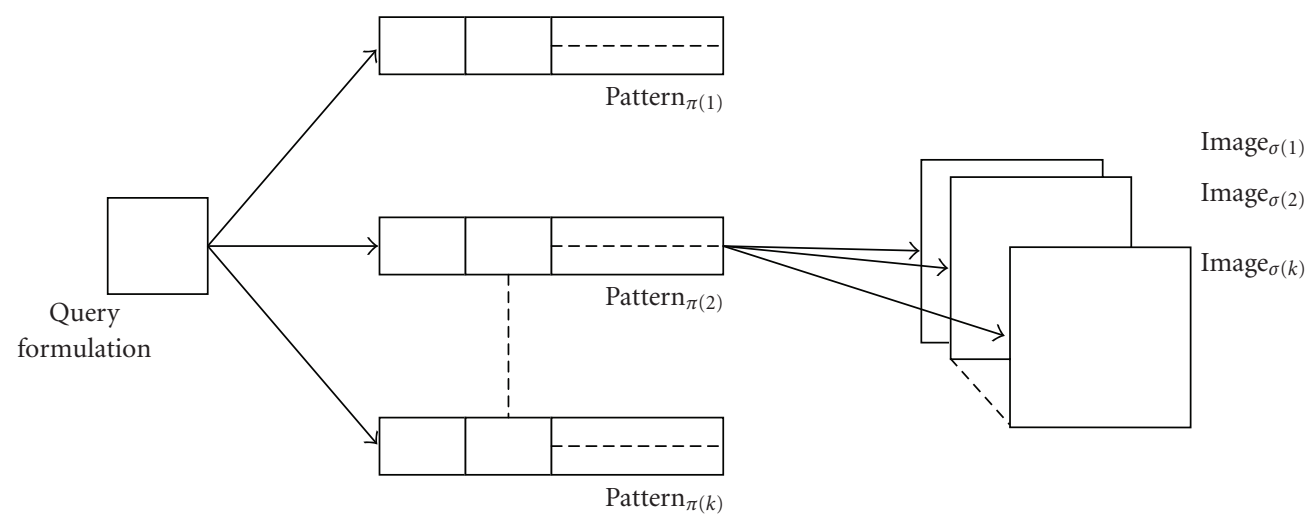

FIGURE 8: Search by pattern example: the system displays pattern ${ }_{\pi(1)}$, pattern $_{\pi(2)} \ldots$, in which the chosen color is the most important, and then the system displays the images in which a selected pattern is the most representative ( $\pi$ and $\sigma$ stand for the permutations that order the patterns and images with respect to the preferred color and pattern, resp.).

which gives the rank- $k$ model with the best possible leastsquares fit to $W_{0}[1]$. The columns of $T$ are the left singular vectors of $W$ and they form a basis for the space spanned by $W$ 's columns; the rows of $D$ are the right singular vectors of $W$ and they are a basis for the space spanned by W's rows.

\section{Color patterns}

Let us consider the interpretation and the advantages of LSI, for our image information extraction purposes. It is important for LSI technique that the derived $W$ matrix does not reconstruct the original bin by image matrix $W_{0}$ exactly. It rearranges the color bin space to reflect the major color association patterns in the data and eliminates the noise and redundancy present in the image data. Since the columns $t^{j}$, $j=1 \cdots k$, of $T$ form a basis for the space spanned by $W$ 's columns, they can be considered the axis of the rearranged space. First axis $t^{1}$ reflects the first major pattern, named patter $_{1}$, present in the image database: $t_{11}$ shows the contribution of $\operatorname{bin}_{1}$ in pattern $1, t_{21}$ shows the contribution of $b_{i n}$ in pattern $_{1}$ and so on up to the last $t_{m 1}$ that shows the contribution of $\operatorname{bin}_{m}$ in the first pattern. Second axis $t^{2}$ reflects the second major pattern in the data, named pattern ${ }_{2}$, and so on up to axis $t^{k}$ corresponding to pattern ${ }_{k}$. Therefore, LSI allows the replacement of individual bins (used initially as descriptors of images) by "independent patterns" that can be specified by any one of several bins or combination of the above mentioned. 


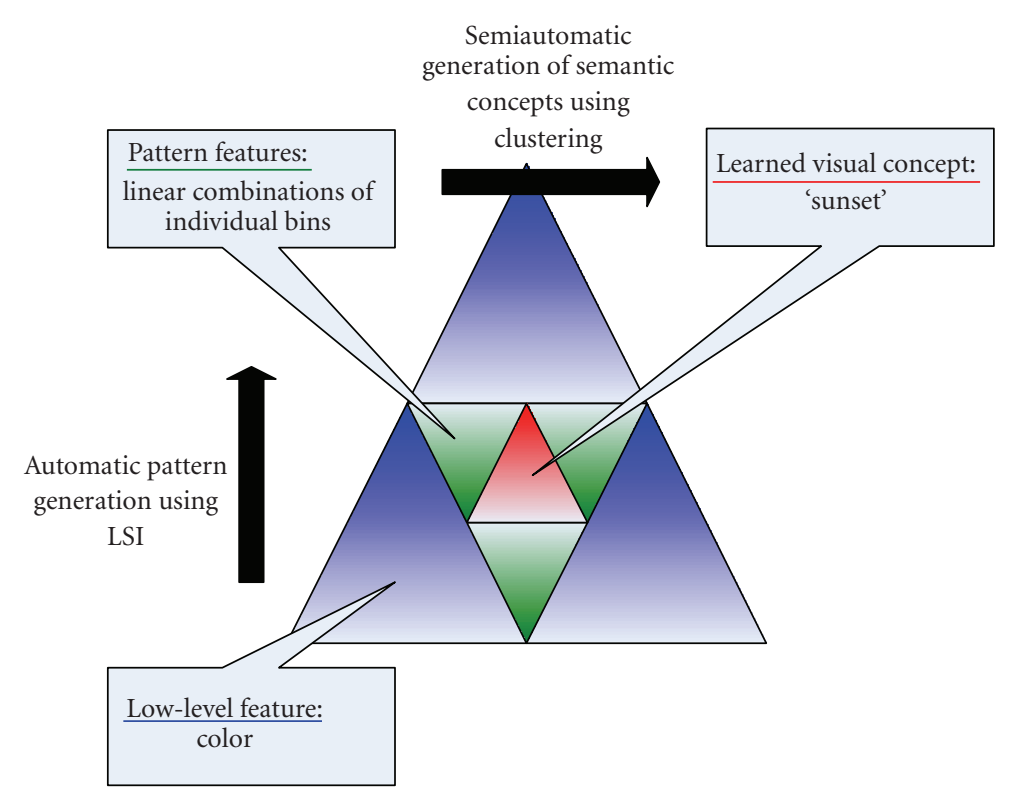

FIGURE 9: The relationship between low-level features, patterns, and high-level concepts can be visualized using the triangle relationship between data, information, and knowledge: patterns (information) are extracted from low-level features (data) and knowledge (learned visual concepts) is discovered from patterns.

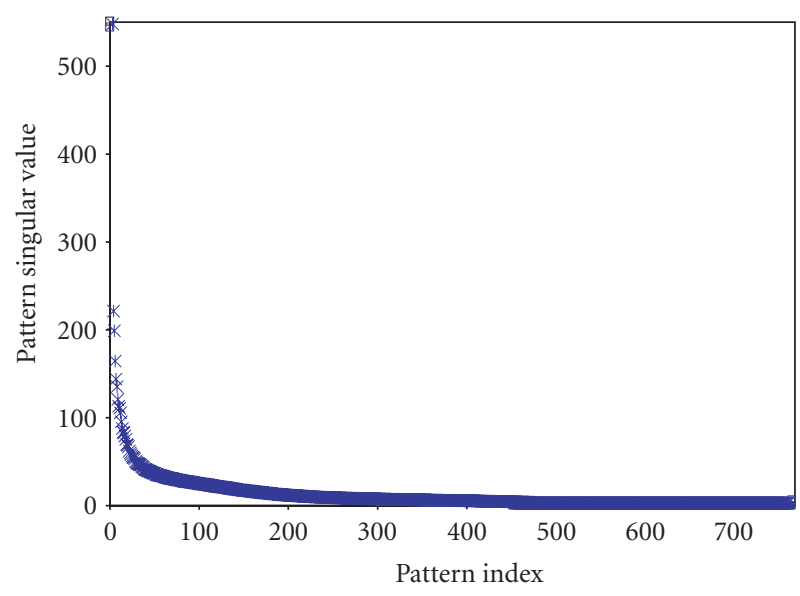

FIGURE 10: Scree plot of the singular values.

Let us consider the new image representations in the pattern space. Using superscript and subscript to denote the columns and rows of a matrix, respectively, and (') to denote the transpose of a matrix, (4) can also be written as

$$
\left(W^{1} W^{2} \cdots W^{n}\right)=\left(T \times \sum\right) \times\left(D^{\prime 1} D^{\prime 2} \cdots D^{\prime n}\right)
$$

where $W^{j}$ stands for the weighted bin representation of $\operatorname{Imag}_{j}, j=1 \cdots n$ and $\left(D^{\prime}\right)^{j}$ is the $j$ th column of matrix $D^{\prime}$. By definition [22], the product between a matrix and a vector is a combination of matrix's columns. Therefore, every $W^{j}$ is a linear combination of the columns of $T \times \sum$ :

$$
W^{j}=\sum_{l=1}^{k} d_{j l}\left(\sigma_{l} t^{l}\right), \quad j=1 \cdots n .
$$

Equation (6) gives the new representation of $\operatorname{Imag}_{j}, j=$ $1 \cdots n$, in which $d_{j 1}, d_{j 2} \cdots d_{j k}$ indicate the strength of association of $\operatorname{Imag}_{j}$ with the discovered pattern $_{1}$, pattern $_{2}$, and pattern $_{k}$, respectively; when images are represented as points in the pattern space, $d_{j 1}, d_{j 2} \cdots d_{j k}$ are the coordinates of the point representing $\operatorname{Imag}_{j}$ in the pattern space whose axes $t^{l}$ are rescaled by $\sigma_{l}, l=1 \cdots k$ (see Figure 4 ).

The derived representation captures most of the important underlying structure in the association of bins and images and at the same time removes the noise and redundancy in the image data. An example of a color pattern composition is presented in Figure 5 and the images in which the corresponding pattern is mostly present are shown in Figure 6.

We would like to point out that the dimension $k$ of the new space represents so-called embedded dimension of the visual data in the original feature space [23]. This dimension should be large enough to fit all the real structure in the data, and thus to yield good retrieval performance, but small enough to not fit the unimportant details. Experimental results presented in Section 4 show that the embedded dimension is significantly smaller than the original dimension, which exactly correlates with White and Jain's affirmations [24].

\section{Synobins generation}

To better understand our model, we will use a series of direct comparisons between LSI for text retrieval and LSI for 


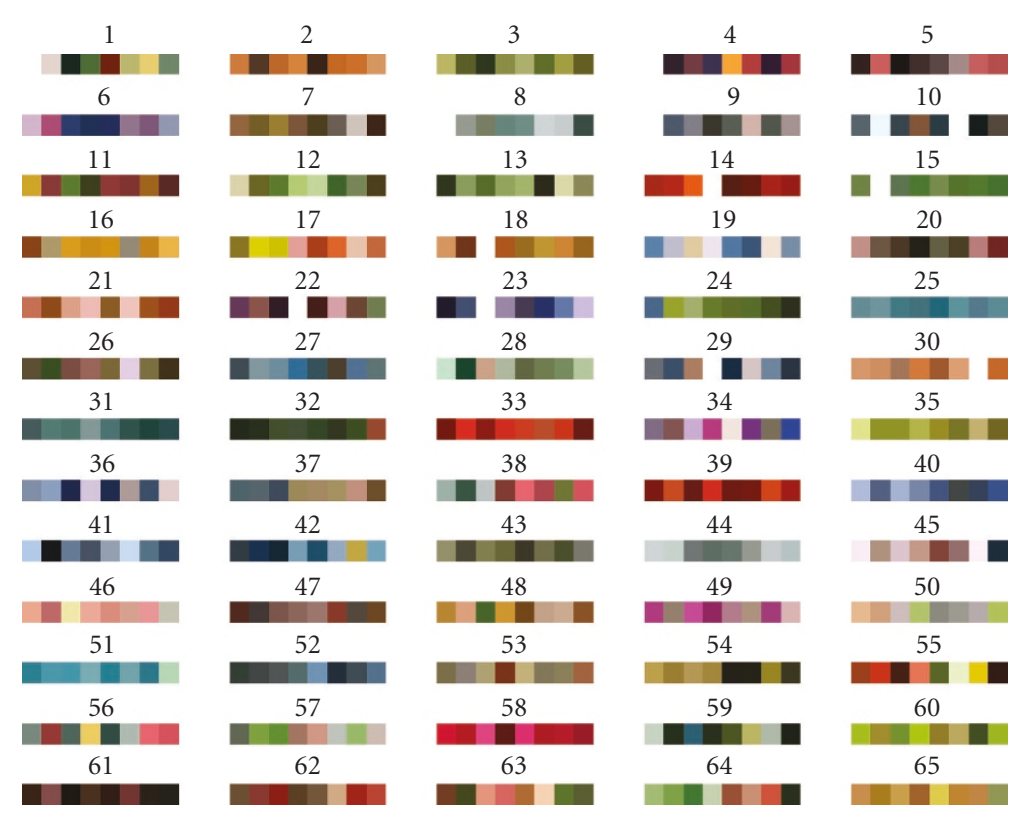

FIGURE 11: The most important 65 color patterns that reflect the color composition of the imaged from the database. The number above the patterns represents the pattern rank, 1 being the most important, and 2 being the second most important, and so on. Just for visualization purposes, each pattern is represented by its most 8 representative color bins (i.e., the most dominant colors within those patterns).

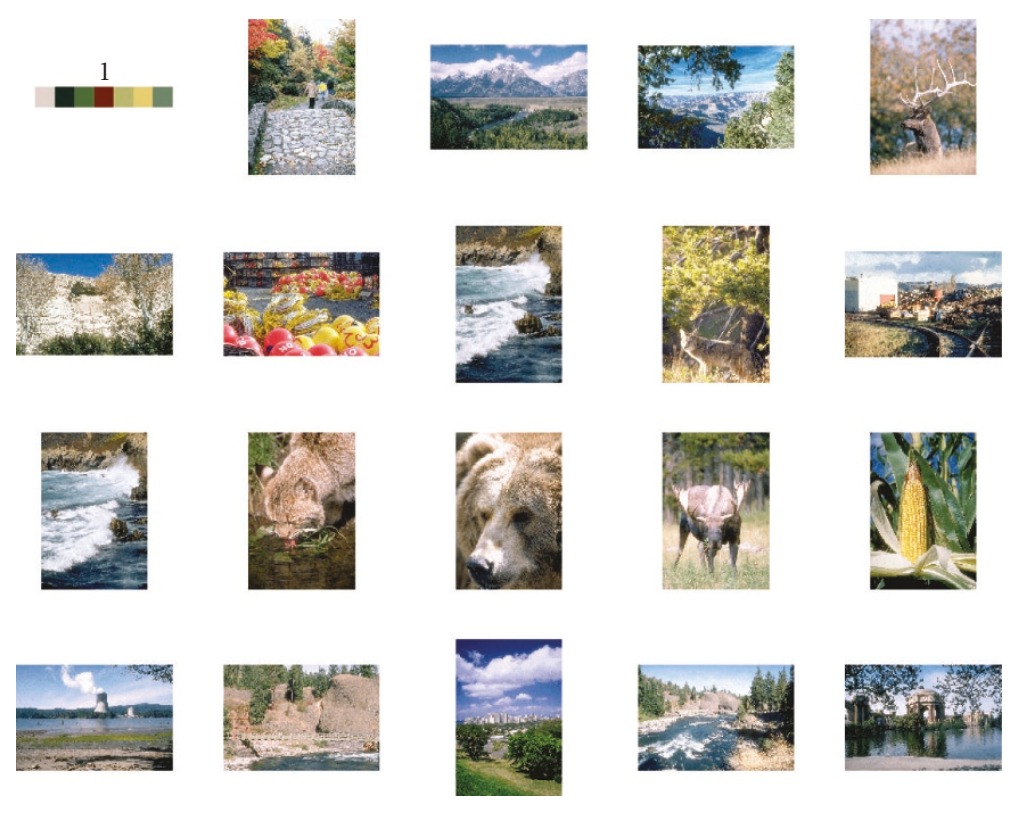

Figure 12: Most representative images for pattern 1.

image retrieval. We coin the term synobin to represent bins that are similarly used across the image database which taken together are the most important ones in defining a pattern. Synobins are directly analogous to synonyms found in text retrieval. The application of LSI in text retrieval allows words synonymous with each other to be near each other in the LSI space; a group of synonym words defines a concept that is later used to perform retrieval by concepts instead of term matching. For example, terms as "physician," "doctor," "surgeon" (spelled different, but with same meaning) will form a concept and the retrieval results for a query based on the term "physician" will also contain documents in which 

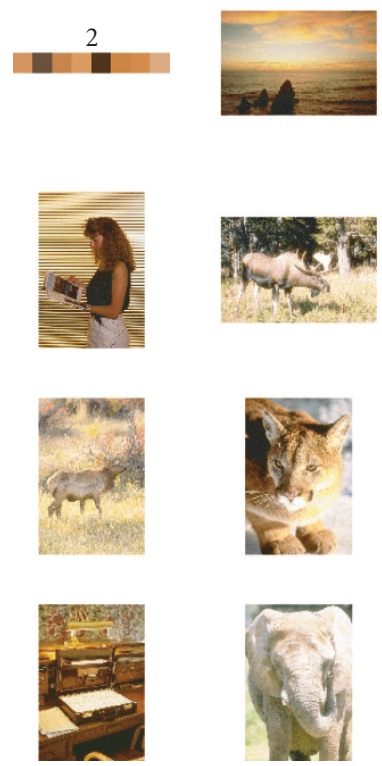
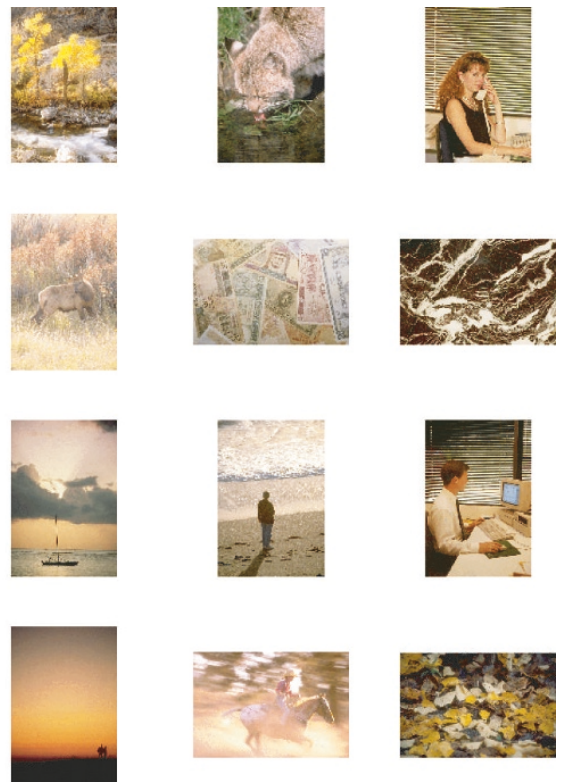

FIGURE 13: Most representative images for pattern 2.
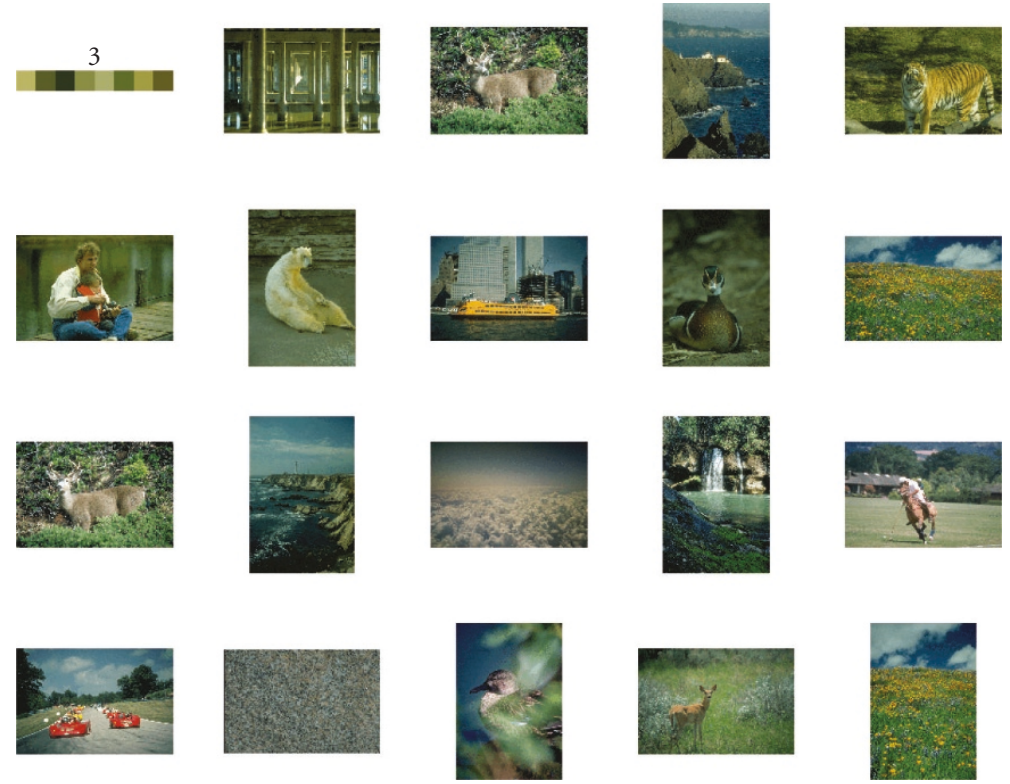

FIGURE 14: Most representative images for pattern 3.

"doctor" or "surgeon" appears even if the "physician" word is not present in those documents. We use the same idea in our image model (Figure 7). Bins representing different colors but similarly used across the image database can be considered as synonymous with each other and the pattern that they define is the analogous of a concept from text retrieval, and thus called synobins.

In the previous subsection, we described the pattern space as a mechanism for finding new representations for images and comparing queries with images. With minor variation, the pattern space can also be used to represent the bins and bin-to-bin comparisons which lead to the discovery of synobins that define interesting patterns.

Recall that the rows of $D$ are the right singular vectors of $W$ and they form a basis for the space spanned by $W$ 's rows. Using (7) (easily obtained from (4)), we claim that this space can also be considered the pattern space since the left and right singular vectors are particular choices of the 


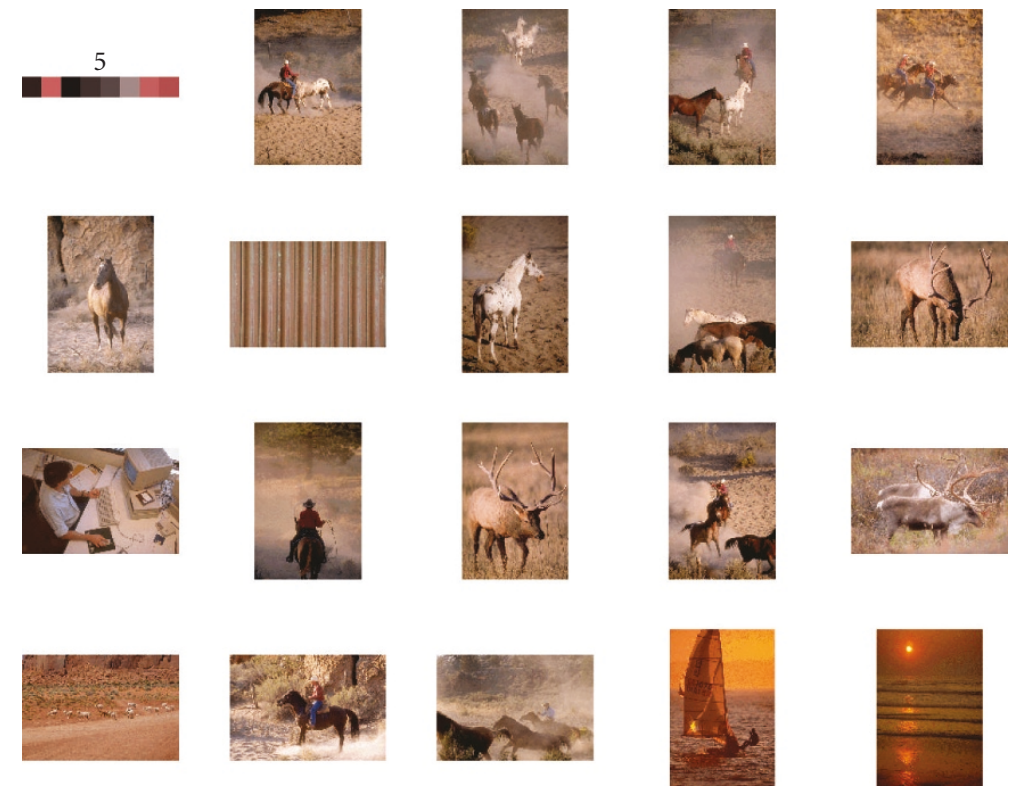

FIGURE 15: Most representative images for pattern 5.
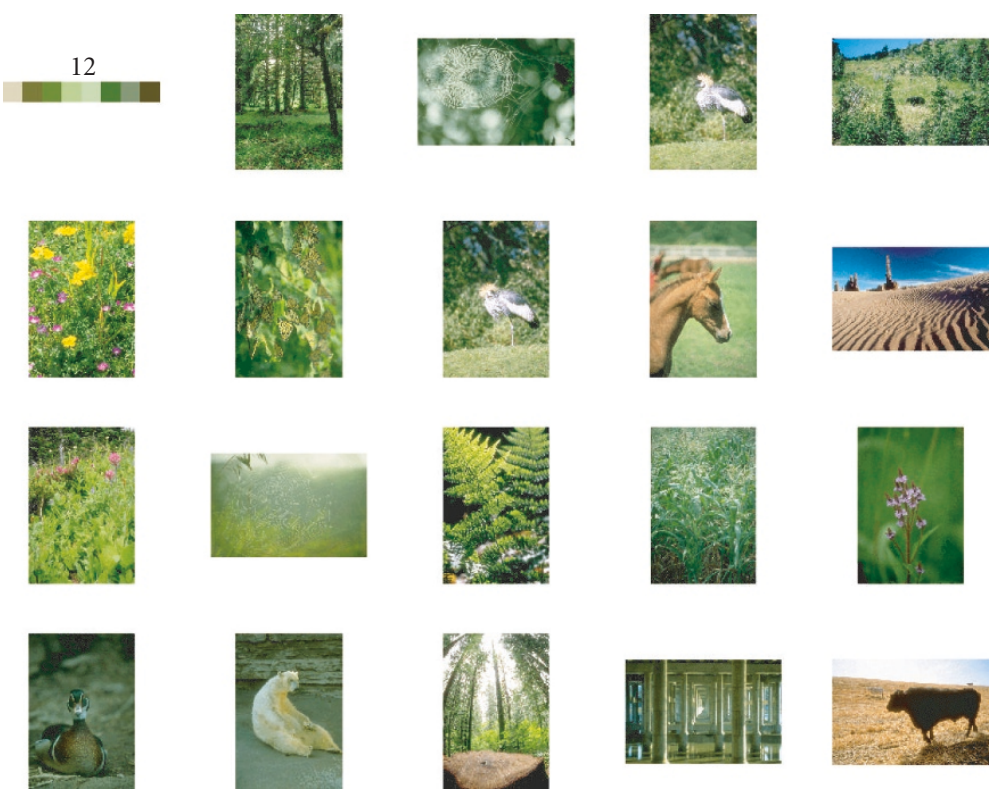

FIGURE 16: Most representative images for pattern 12.

eigenvectors of $W \times W^{\prime}$ and $W^{\prime} \times W$, and these eigenvectors correspond to the same set of eigenvalues $\sqrt{\sigma_{i}}, i=1 \cdots k$ :

$$
\begin{aligned}
& W^{\prime} \times W=D \times \sum^{2} \times D^{\prime}, \\
& W \times W^{\prime}=T \times \sum^{2} \times T^{\prime} .
\end{aligned}
$$

Following the same rationale as for the new image representation, the bin representation for $\operatorname{bin}_{i}$ in the pattern space will be given by

$$
W_{i}=\sum_{l=1}^{k} t_{i l}\left(\sigma_{l} d_{l}\right), \quad i=1 \cdots m
$$

The coefficients $\left(t_{i 1}\right), l=1 \cdots k, i=1 \cdots m$, can be interpreted in two ways.

(i) When $l$ is fixed, a descending order for $t_{1 l}, t_{2 l}, \ldots, t_{m l}$ will reveal the synobins (the bins on the first positions) defining the $\operatorname{pattern}_{l}$. 


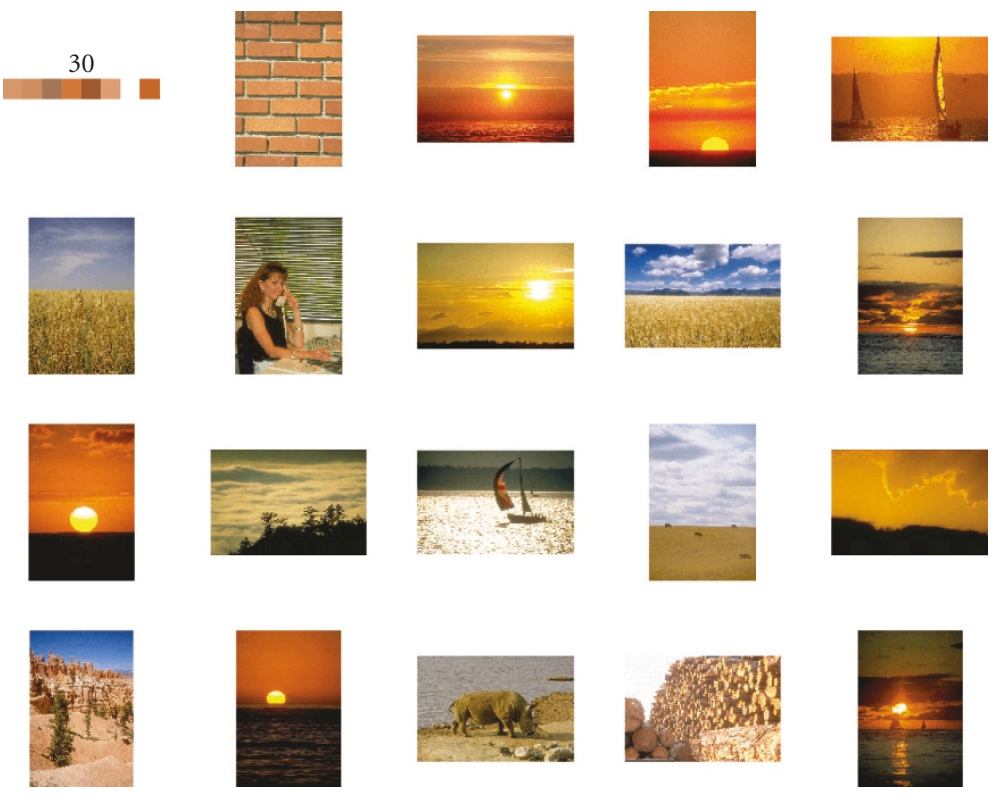

FIgURE 17: Most representative images for pattern 30.
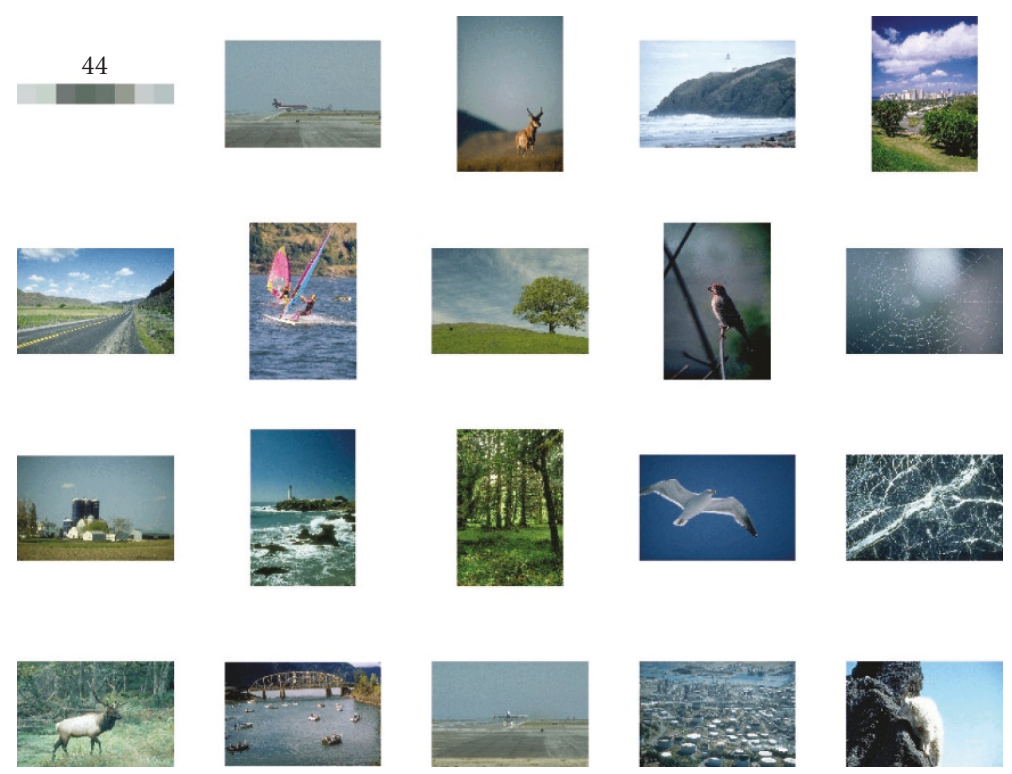

FIgURE 18: Most representative images for pattern 44.

(ii) When $i$ is fixed, a descending order for $t_{i 1}, t_{i 2}, \ldots, t_{i k}$ will reveal the patterns in which $\operatorname{bin}_{i}$ is mostly present.

\section{SYNOBINS AND ACROSS-IMAGE CONTEXTS FOR IMAGE ANNOTATION}

Summarizing the image LSI model, we obtained a common ground for images and features, specifically the lower $k$ dimensional pattern space. In this space, both images and bins are uniformly represented as points, and if there are any groups of bins and images in this space, they will be considered as contexts for the image database. More exactly, the group of bins placed near each other will form a within-image context and it is most likely that they are the most important bins in forming an interesting pattern. The points representing images that belong to the same group will form an across-image context; if these images have the same semantic interpretation, that semantic meaning will 


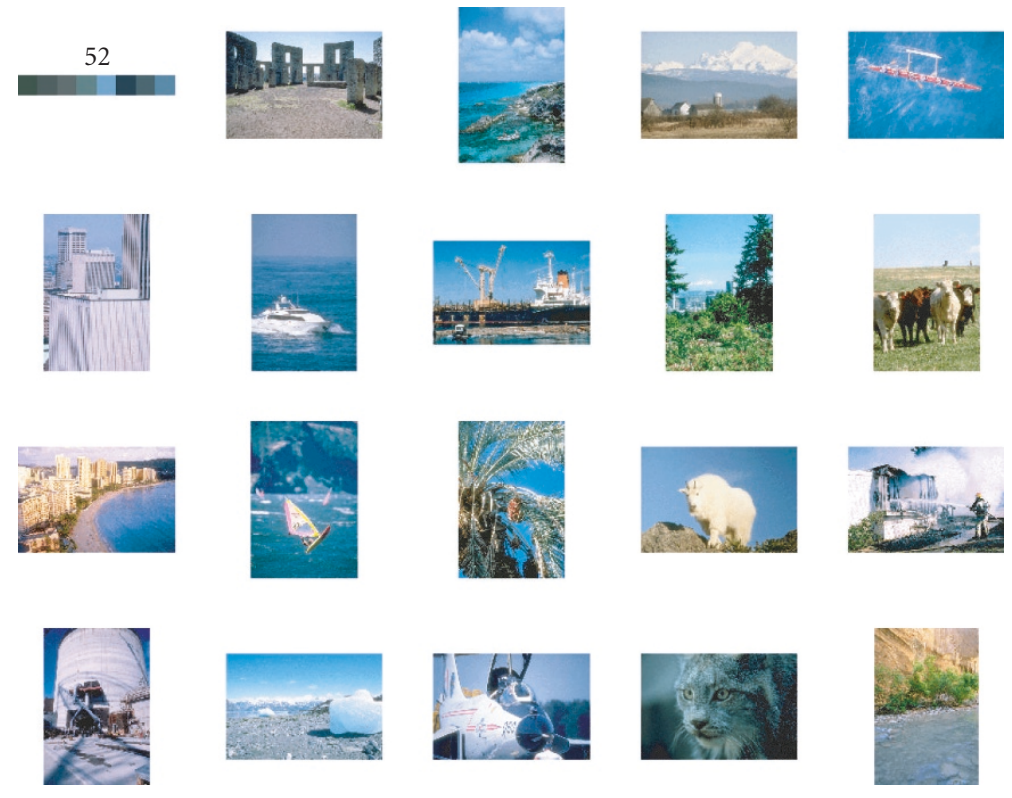

FIGURE 19: Most representative images for pattern 52.

be associated to the color pattern, and thus to the synobins forming that pattern. To understand better, picture the above ideas for document retrieval. The word "doctor" is present in a group of documents about medical field along with some other words such as "physician" and "surgeon." From the context in which the word is used, the meaning of the word "doctor" will be associated with a person licensed to practice medicine and not with a person who has been awarded a higher academic degree in any field of knowledge, such as "doctorate."

There are two scenarios.

(i) Textual information (keywords, labels) is provided for images to describe their visual content. In this case, the most frequent keyword in the corresponding group of image points will be considered the semantic interpretation.

(ii) There is no textual information provided with the images. In this case, our system acts like a semiautomatic tool for annotation: different groups of synobins and similar images are presented to the person who is in charge with the annotation. The advantage is that the annotator does not have to search the whole database to look for similar images with the image that has to be annotated. The group of images and bins in which the image appears will offer a context in which the image can be interpreted and annotated.

We propose clustering approach to perform the process of grouping bins and images in the pattern space. We do not present the mathematics and advantages of the clustering approach for our system. For details, we refer the reader to $[25,26]$, where we used a hierarchy of clusters to

(i) provide a semiautomatic tool for image annotation;

(ii) allow browsing through a large image database, going from finer to finer details.
In addition to the above advantages, the clustering approach offers an additional functionality to our system. It allows the capability of learning associations between not only semantic concepts and images, but also between semantic concepts and patterns. This is very important when a user does not look for a particular image, but for a pattern of colors that will be present in the image. Think about a web designer who is searching an image database to find a background image to use for a page. He does not have in mind any conceptualization of the image that he is looking for, what he knows is that the background should have some particular color or combination of colors in it. Our system can prompt the designer to identify which of the different patterns contain the color of interest, and thus it helps the designer to refine the search.

Another significant advantage to having a CBIR system based on synobins is the improved efficiency of the retrieval process while still yielding excellent semantically related results; based on our experimental results, we found that merely $3 \%$ of the original bins are enough to obtain good retrieval results. Furthermore, the clustering approach allows the search space to be reduced to the square root of the original search space; a more complex hierarchical clustering technique could further reduce the search space logarithmically.

\section{IMAGE RETRIEVAL IN THE CONTEXT}

A CBIR system having the pattern extraction component incorporated allows mainly two types of image database search.

(i) Search by image example: the image query is represented in the pattern space and the process of searching for similar images is based on pattern similarity instead of individual bin similarity. We refer to this retrieval as image 

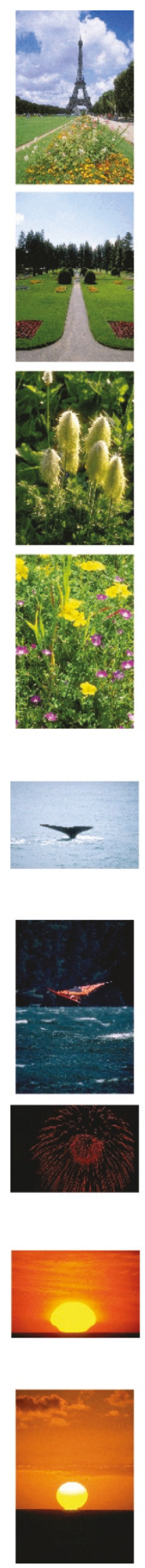
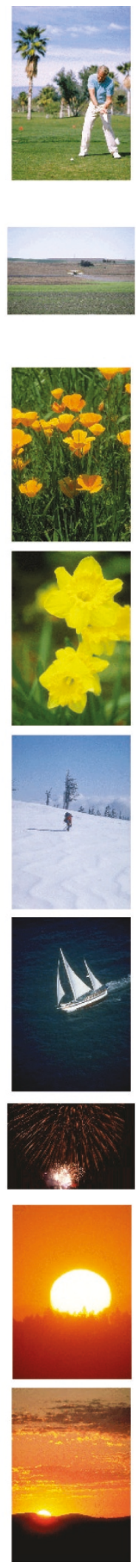
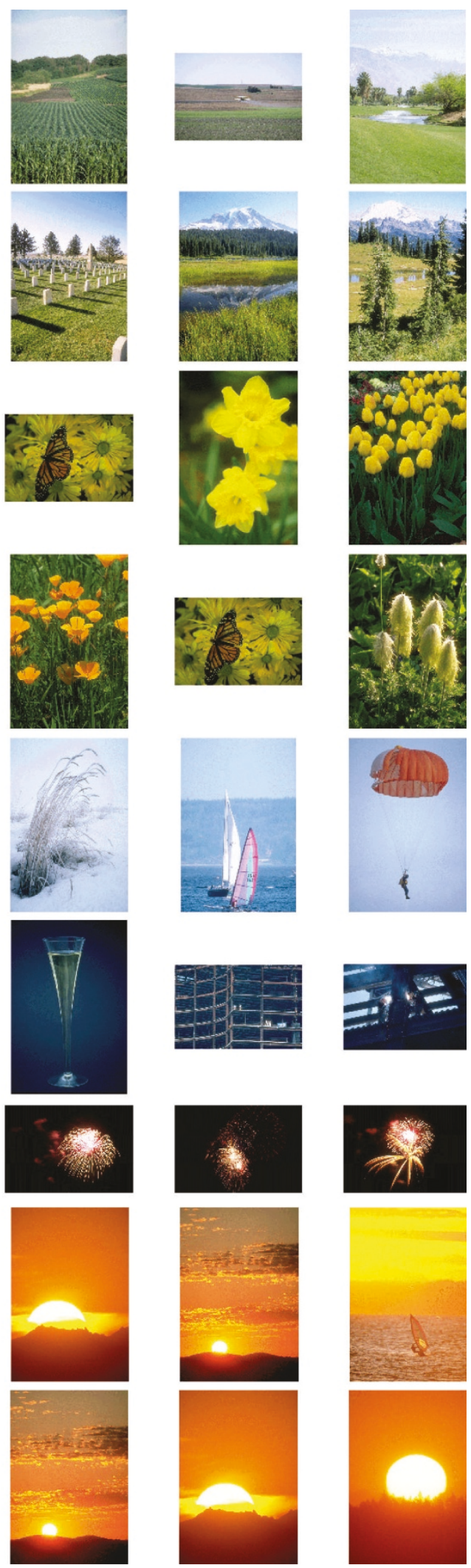

FIGURE 20: Retrieval results for several query images; the first image on each row represents the query image and the next images on each row represent the most similar images with the query image in the order of their similarity. 
TABle 1: Percentage of variance captured by the singular values (patterns).

\begin{tabular}{lccc}
\hline No. of patterns & Variance $(\%)$ & No. of patterns & Variance $(\%)$ \\
\hline 1 & 7.12 & 273 & 92.59 \\
33 & 41.99 & 305 & 94.52 \\
65 & 56.07 & 337 & 96.13 \\
81 & 61.60 & 369 & 97.47 \\
113 & 70.90 & 401 & 98.56 \\
145 & 78.09 & 433 & 99.39 \\
177 & 83.42 & 465 & 99.90 \\
209 & 87.31 & 481 & 100 \\
241 & 90.24 & 768 & 100 \\
\hline
\end{tabular}

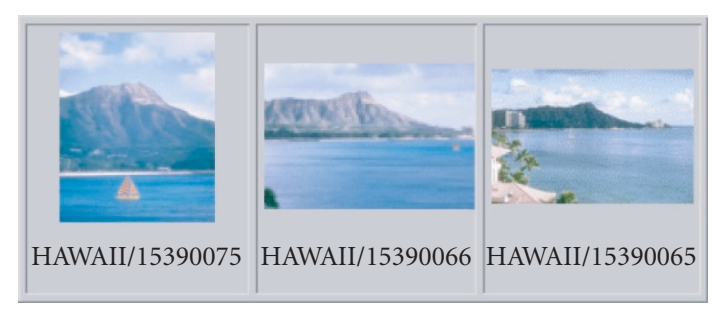

Figure 21: The most similar two images with the query image.

retrieval in the context: even if an image is not indexed by the query low-level features, if it shares synobins with another image that is relevant to the given query, it is likely to be retrieved.

(ii) Search by pattern example: the most important patterns in the database are presented to the user. The user selects a pattern of interest, and then the system provides the images in which the pattern is mostly present (Figure 8). Furthermore, the user chooses one of interest from these images and the system retrieves most similar images with the selected one.

The search-by-pattern example moves the CBIR system beyond simple matching of images based on primitive features. Thinking about patterns as a new type of image feature (Figure 9) situated between the low-level features and the high-level ones (keywords, annotations, etc.), our model provides information about which low-level feature elements are most important and how they should be combined for a given pattern and which patterns are most important for a given annotation.

This makes our CBIR system an important step towards semantic retrieval.

The issue of choosing a similarity metric to measure the similarity between images is outside the scope of this paper; however, since measuring meaningful image similarity is based not only on finding the right image features but also on choosing the right metric for the feature space, we will expand on it in future work. As a quick reference, we used cosine measure and histogram intersection to implement our system. The reason behind choosing cosine measure was that it is the natural measure of similarity between two vectors in the pattern space induced by SVD. If $q_{0}$ is a query image and $q=q_{0} \times T \times \sum^{-1}$ is its reduced representation [3] in the $k$ dimensional pattern space, the cosine similarity between the query $q$ and an image $\operatorname{Imag}_{j}, j=1 \cdots n$, is defined as

$$
\operatorname{sim}\left(\operatorname{Imag}_{j}, q\right)=\frac{\sum_{l=1}^{k}\left(q_{l} * d_{j l}\right)}{\left(\sum_{l=1}^{k} q_{l}^{2}\right)^{1 / 2} *\left(\sum_{l=1}^{k} d_{j l}^{2}\right)^{1 / 2}}
$$

in which $\left(d_{j 1}, d_{j 2} \cdots d_{j k}\right)$ represents the reduced image representation of $\operatorname{Imag}_{j}$.

It is worth to mention here that if one decides to apply the histogram intersection method [19], the $L_{1}$-form has to be used:

$$
\operatorname{sim}\left(\operatorname{Imag}_{j}, q\right)=\left(\frac{1}{2 * k}\right) * \sum_{l=1}^{k}\left|d_{j, l}-q_{l}\right|
$$

The choice of the $L_{1}$-form is motivated by the fact that some of the values from the pattern representations might have negative values and for such values, the fast incremental histogram intersection version does not make too much sense. The presence of negative elements in $d_{l j}, l=1 \cdots k$, is not a problem but rather a reflection of the fact that the patterns are linear combinations of individual bins; the image database is modeled by the geometric relationships between image vectors and not by individual components of these vectors [27].

\section{EXPERIMENTAL RESULTS}

\section{Image database and color space}

In this section, we present the preliminary results obtained in the evaluation of our approach. We implemented our system using a general-purpose database of 2100 RGB images. First, we transformed the images into HSV color space, and then we calculated separately three 256-bin global histograms (for hue, saturation, and value) for each image. The SVD code from Matlab provided us with the set of singular values already arranged in sorted order. This is very convenient to us, because when we vary the number $k$ of singular values to obtain our reduced pattern representation, we want to pick the largest singular values such that the noise and redundancy are eliminated and the best retrieval performance is obtained. 


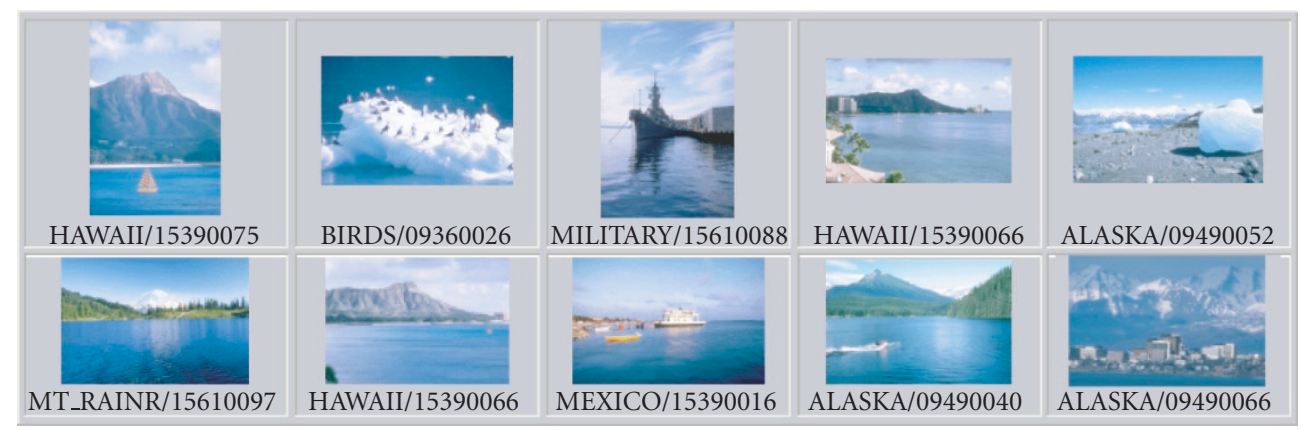

FIGURE 22: Images retrieved using histogram intersection on global histograms.

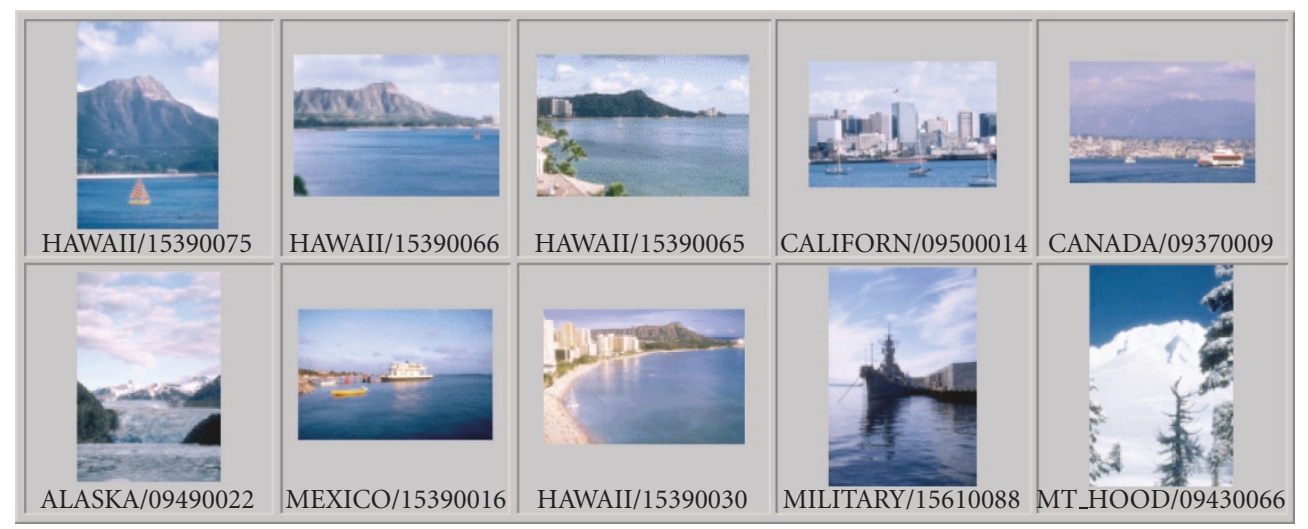

FIgURE 23: Images retrieved when cosine measure is used and $k=20$.

The matrix $t$ is of size $768 \times 768$ (768 patterns which are linear combinations of 768 bins), the matrix $s$ is a diagonal matrix of size $768 \times 768$ (the $i$ th element on the diagonal represents the $i$ th singular value for pattern $\# i$ ), and the matrix $d$ is of size $768 \times 2100$ (each of the 2100 documents is represented with respect to the 768 patterns).

\section{Color patterns and Synobins Generation}

Looking at the plot (Figure 10) of the 768 singular values calculated for the 2100 color image database, we noticed that the first 240 singular values are greater than zero and they also capture more than $90 \%$ (Table 1) of the color variance in the database; in other words, by using only 250 color patterns instead of 768 bins, one would still be able to capture over $90 \%$ of the original color structure present in the images.

Furthermore, the first 481 values are enough to capture the entire color structure of the database, so the other patterns can be neglected without losing any color information. Figure 11 shows the first 65 patterns from the database that capture over $50 \%$ of the color patterns in the image database and it can be used as a starting point for doing search by pattern as described in Section 5; the user can select any of the patterns presented below and then the system will return the images in which the selected pattern is mostly present. For an illustration of the search-by-pattern results, we present the most 19 representative images for several patterns in the database; these patterns were chosen such that we show a variety of color patterns along with their corresponding images (Figures 12, 13, 14, 15, 16, 17, 18, and 19).

It is also interesting to notice the latent correlation between the new features (color patterns) and the semantic concepts associated with each cluster of images retrieved for each pattern. When most of the images share the same semantic concepts, then those semantic concepts can be associated with the patterns and also with the images they represent. For example, pattern \#1 and its most representative images can be associated with the concept "landscape," where landscape is denoted by a combination of the following terms: sea, sky, grass, mountains, and land; pattern \#5 and its images can be associated with either one of the following concepts: arid, texture, or sunset.

\section{Retrieval by color patterns}

Once the user decides that he is interested in a certain image, then that image becomes an image query and using a similarity metric, the system will retrieve the most similar images with the query image based on pattern similarity. Figure 20 shows examples of retrieval results for several query images where only 20 patterns were used to perform the retrieval.

In evaluating the retrieval performance of our approach, we focus only on the local topology of the pattern space and 


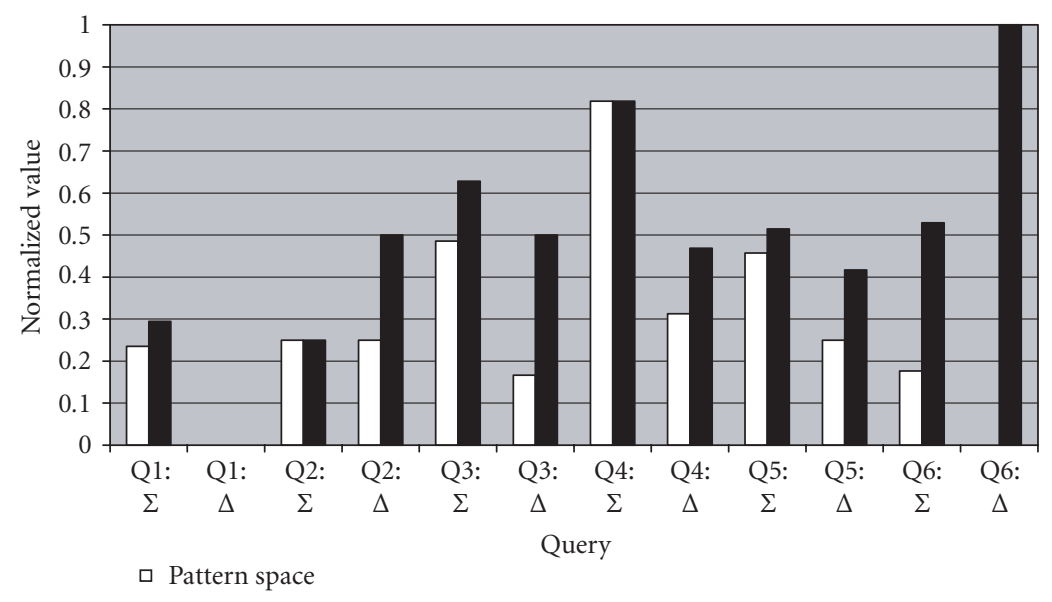

- Histogram space

Figure 24: Perceptual accuracy evaluation as calculated with formulas (11) and (12).

not on the used similarity metric since we want to see if better perceptual similarity of images can be obtained from the different nature of the new feature space itself. Our current results are produced using the cosine similarity measure as being the most-used similarity measure in the vector space and using the histogram intersection as being the most used for color histogram similarity. In a future implementation of our approach, we plan to incorporate other similarity measures that will take into account the human perception in evaluating the retrieval results; one of such measures is the Color-WISE metric that we previously used to build a hierarchy of clusters for color image databases [25]. Furthermore, in order to evaluate the retrieval performance, as a ground truth, for each query, a set of most similar images is assigned together with a relative ranked order of their similarity.

\section{Evaluation of the retrieval}

We present the results for a query image (left image from Figure 21) belonging to a "landscape" image category from the database. We define an image as being "landscape" if it contains sky, water, and land. The image database contains 98 images of this category and the second and third images from Figure 21 represent the ground truth in measuring the retrieval accuracy for the chosen query image $q$.

Comparisons between the results of histogram intersection in the original 768-dimensional space (Figure 22) and the results of cosine measure in the pattern space (Figure 23) show that even if the feature space was reduced to only 20 features, the retrieval accuracy is the same as for the original space. Moreover, if the users are asked to evaluate the performance, the retrieval results from Figure 23 are more perceptual appealing then those from Figure 21 because the images considered as the ground truth appear closer to the query image in Figure 23 than those in Figure 22 do.

That is, using color patterns, the images considered as ground truth appear as the first and second best matches (not considering the query image itself), while using global histograms, same images appear as the third and sixth best matches. This observation is very important in the context of image retrieval where we are interested in displaying only the top few retrieval results. For example, if the system had been designed to display, let us say, only the first two retrieved images, no images from the ground truth would have been displayed to the user in the case of global histogram.

In order to quantify the perception issue discussed above, let $G T(q)$ be the ground truth ordered with respect to human perception and let $R(q)=\left\{N_{1}, \ldots, N_{P}\right\}$ be the set of the nearest neighbors ordered with respect to a defined similarity measure (cosine or histogram intersection). The parameter $\sigma(q)$ in formula (11) measures how close the most relevant images with the query image are situated in the list $R(q)$ of the nearest neighbors and a lower value of the parameter indicates a better retrieval result:

$$
\sigma(q)=\sum_{s_{i} \in R(q) \cap G T(q)} \operatorname{rank}\left(N_{i}, R(q)\right) .
$$

The $\operatorname{rank}(\cdot, \cdot)$ notation gives the position of an element in a list and is defined as $\operatorname{rank}(N, R(q))=i$, whenever $N=N_{i}$. Moreover, if $\sigma(q)$ is the same in pattern space and original space, an additional objective measure $\Delta(q)$ defined in formula (12) can be considered to quantify the relative positions of the most relevant images and a lower value of $\Delta(q)$ indicating a better retrieval:

$$
\Delta(q)=\sum_{\substack{i=1 \\ N_{i} \in R(q) \cap G T(q)}}^{L(q)}\left|i-\operatorname{rank}\left(N_{i}, G T^{\prime}(q)\right)\right|,
$$

where $L(q)$ is the cardinality of $G T(q) \cap R(q)$ and $G T^{\prime}(q)$ is the set $G T(q) \cap R(q)$ ordered with respect to human perception (a subset of the ground truth).

Figure 24 gives the normalized values of $\sigma(q)$ and $\Delta(q)$ for landscape queries, for which the precision and recall are the same, and therefore, we calculated sigma $(\sigma(q))$ and delta $(\Delta(q))$ in order to evaluate the overall accuracy for every space. 


\section{SUMMARY AND FUTURE WORK}

In this paper, we proposed patterns of colors and intensities as image features, which are meant to capture the latent associations among images and primitive features in such a way that the noise and redundancy are eliminated. We introduced the synobin, a new term for content-based image retrieval literature, which is the equivalent of a synonym word from text retrieval, to name the bin that is synonymous with other bins of a color feature, in the sense that they are similarly used across the image database. Incorporating our feature model into a CBIR system moves the research in image retrieval beyond simple matching of images based on their primitive features and creates a ground for learning image semantics from visual content. Explicitly, it will be useful in developing new CBIR systems having the following characteristics.

(i) Retrieval in the context: images are retrieved based on patterns of colors and intensities present in the images and not by matching individual bins.

(ii) Semiautomatic image and pattern annotation: instead of giving all possible meanings to an image, the system interprets an image based on the color patterns and the acrossimage context in which it appears; if there is a semantic concept associated with the across-image context, that concept can be also associated with the corresponding patterns.

We evaluated the performance of our system based on the retrieval accuracy and on the perceptual similarity order among retrieved images. When compared to standard image retrieval methods, our preliminary results show that even if the feature space was reduced to 20-30 features (out of 768), the accuracy and perceptual similarity for our system remain the same or better depending on the category of images. Since the used similarity metrics did not take into account the human perception, we believe that the better perceptual similarity of images might come from the different nature of the new feature space.

A complete characterization of the time performance goes beyond this paper, but we make few remarks here. The use of the hierarchy of clusters, which will be a more efficient indexing tool, along with a branch-and-bound algorithm for a fast calculation of the nearest neighbors, will all speed up the retrieval process. With respect to the time of calculating the new patterns, we will pursue future work in applying faster algorithms to calculate and update the singular value decomposition. Furthermore, we will experiment the integration of color, shape, and structural image in order to produce a unified new feature space using latent semantic indexing; moreover, we will test the new proposed approach over various large image databases used as benchmarks in the image retrieval research community.

\section{REFERENCES}

[1] S. Santini and R. Jain, "Similarity is a geometer" Multimedia Tools and Applications, vol. 5, no. 3, pp. 277-306, 1997.

[2] A. Guttman, "R-trees: a dynamic index structure for spatial searching," in Proceedings of ACM SIGMOD International Conference on the Management of Data (SIGMOD '84), pp. 4757, Boston, Mass, USA, June 1984.
[3] S. Deerwester, S. T. Dumais, G. W. Furnas, T. K. Landauer, and R. Harshman, "Indexing by latent semantic analysis," Journal of American Society for Information Science, vol. 41, no. 6, pp. 391-407, 1990.

[4] M. Flickner, H. Sawhney, W. Niblack, et al., "Query by image and video content: the QBIC system," IEEE Computer, vol. 28, no. 9, pp. 23-32, 1995.

[5] J. R. Bach, C. Fuller, A. Gupta, et al., "Virage image search engine: an open framework for image management," in Storage and Retrieval for Still Image and Video Databases IV, vol. 2670 of Proceedings of SPIE, pp. 76-87, San Jose, Calif, USA, February 1996.

[6] J. Feder, "Towards image content-based retrieval for the world-wide web," Advanced Imaging, vol. 11, no. 1, pp. 26-29, 1996.

[7] J. R. Smith and S.-F. Chang, "VisualSEEk: a fully automated content-based image query system," in Proceedings of 4th ACM International Multimedia Conference (ACM Multimedia '96), pp. 87-98, Boston, Mass, USA, November 1996.

[8] M. Ortega, Y. Rui, K. Chakrabarti, S. Mehrotra, and T. S. Huang, "Supporting similarity queries in MARS," in Proceedings of 5th ACM International Multimedia Conference (ACM Multimedia '97), pp. 403-413, Seattle, Wash, USA, November 1997.

[9] W. Y. Ma and B. S. Manjunath, "NeTra: a toolbox for navigating large image databases," in Proceedings of IEEE International Conference on Image Processing (ICIP '97), vol. 1, pp. 568-571, Santa Barbara, Calif, USA, October 1997.

[10] J. P. Eakins and M. E. Graham, "Content-based image retrieval: a report to the JISC technology applications programme," Tech. Rep., Institute for Image Data Research, University of Northumbria, Newcastle, Tyneside, UK, 1999.

[11] M. La Cascia, S. Sethi, and S. Sclaroff, "Combining textual and visual cues for content-based image retrieval on the World Wide Web," in Proceedings of IEEE Workshop on Content-Based Access of Image and Video Libraries (CBAIVL '98), pp. 24-28, Santa Barbara, Calif, USA, June 1998.

[12] T. Westerveld, D. Hiemstra, and F. de Jong, "Extracting bimodal representations for language-based image retrieval," in Proceedings of Multimedia '99, Proceedings of the Eurographics Workshop, pp. 33-42, Milano, Italy, September 1999.

[13] M. Petrou and P. Bosdogianni, Image Processing: The Fundamentals, John Wiley \& Sons, New York, NY, USA, 1999.

[14] L. Zhiu, A. Rao, and A. Zhang, "Theory of keyblock-based image retrieval," ACM Transactions on Information Systems, vol. 20, no. 2, pp. 224-257, 2002.

[15] L. Zhu, A. Rao, and A. Zhang, "Advanced feature extraction for keyblock-based image retrieval," Information Systems, vol. 27, no. 8, pp. 537-557, 2002.

[16] T. P. Minka and R. W. Picard, "Interactive learning using a society of models," Tech. Rep. 349, MIT Media Laboratory Perceptual Computing Section, Cambridge, Mass, USA, 1995.

[17] C. W. Niblack, R. Barber, W. Equitz, et al., "QBIC project: querying images by content, using color, texture, and shape," in Storage and Retrieval for Image and Video Databases, vol. 1908 of Proceedings of SPIE, pp. 173-187, San Jose, Calif, USA, January-February 1993.

[18] D. Stan and I. K. Sethi, "Color patterns for pictorial content description," in Proceedings of ACM Symposium on Applied Computing (SAC '02), pp. 693-698, Madrid, Spain, March 2002.

[19] M. J. Swain and D. H. Ballard, “Color indexing," International Journal of Computer Vision, vol. 7, no. 1, pp. 11-32, 1991.

[20] I. K. Sethi, I. L. Coman, B. Day, et al., "Color-WISE: a system for image similarity retrieval using color," in Storage and 
Retrieval for Image and Video Databases VI, vol. 3312 of Proceedings of SPIE, pp. 140-149, San Jose, Calif, USA, January 1998.

[21] R. Baezo-Yates and B. Ribeiro-Neto, Modern Information Retrieval, Addison-Wesley-Longman, Boston, Mass, USA, 1999.

[22] L. N. Trefethen and D. Bau III, Numerical Linear Algebra, SIAM, Philadelphia, Pa, USA, 1997.

[23] Y. Rui, T. S. Huang, and S.-F. Chang, "Image retrieval: past, present, and future," Journal of Visual Communication and Image Representation, vol. 10, pp. 1-23, 1999.

[24] D. A. White and R. Jain, "Similarity indexing: algorithms and performance," in Storage and Retrieval for Still Image and Video Databases IV, vol. 2670 of Proceedings of SPIE, pp. 62-73, San Jose, Calif, USA, February 1996.

[25] D. Stan and I. K. Sethi, "Image retrieval using a hierarchy of clusters," in Proceedings of 2nd International Conference on Advances in Pattern Recognition (ICAPR '01), pp. 377-386, Rio de Janeiro, Brazil, March 2001.

[26] D. Stan and I. K. Sethi, "Mapping low-level image features to semantic concepts," in Storage and Retrieval for Media Databases, vol. 4315 of Proceedings of SPIE, pp. 172-179, San Jose, Calif, USA, January 2001.

[27] M. W. Berry, Z. Drmac, and E. R. Jessup, "Matrices, vector spaces, and information retrieval," SIAM Review, vol. 41, no. 2, pp. 335-362, 1999.

Daniela Stan Raicu is an Assistant Professor in School of Computer Science, Telecommunications, and Information Systems at DePaul University in Chicago. She received her B.S. degree in mathematics from University of Bucharest, Romania, in 1993, M.A. degree in computer science from Wayne State University, Michigan, in 1999, and her Ph.D. degree in computer science from Oakland University, Michigan, in 2002. Her research interests include medical imaging, multimedia indexing and retrieval, pattern recognition, data mining, and knowledge discovery. Daniela has published numerous papers in her research areas, received National Science Foundation (NSF) funding to support medical informatics undergraduate research, and is actively involved in organizing different conferences and workshops. She is a Member of the ACM, IEEE, and SPIE. Prior to joining DePaul University, she worked at Accenture Technology Labs, Ford Motor Company, and the Institute of Mathematics in Bucharest, Romania.

Ishwar K. Sethi received the Ph.D. degree from Indian Institute of Technology, Kharagpur, India, in 1978. In 1982, he joined Wayne State University where he was involved in teaching and research in artificial neural networks, pattern recognition, computer vision, and multimedia information retrieval. Since August 1999, he has been with Oakland University where he is the Chair of Computer Science and Engi-

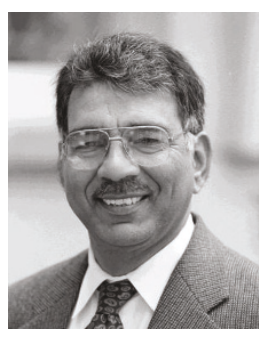
neering Department. Professor Sethi has served on numerous program committees of national and international conferences as well as on editorial boards of many academic journals including IEEE Transactions PAMI, IEEE Multimedia, Pattern Recognition, Pattern Recognition Letters, and Machine Vision and Applications. He was elected IEEE Fellow in 2001. 


\section{Special Issue on}

\section{Search and Retrieval of 3D Content and Associated Knowledge Extraction and Propagation}

\section{Call for Papers}

With the general availability of 3D digitizers, scanners, and the technology innovation in $3 \mathrm{D}$ graphics and computational equipment, large collections of 3D graphical models can be readily built up for different applications (e.g., in CAD/CAM, games design, computer animations, manufacturing and molecular biology). For such large databases, the method whereby 3D models are sought merits careful consideration. The simple and efficient query-by-content approach has, up to now, been almost universally adopted in the literature. Any such method, however, must first deal with the proper positioning of the $3 \mathrm{D}$ models. The two prevalent-in-the-literature methods for the solution to this problem seek either

- Pose Normalization: Models are first placed into a canonical coordinate frame (normalizing for translation, scaling, and rotation). Then, the best measure of similarity is found by comparing the extracted feature vectors, or

- Descriptor Invariance: Models are described in a transformation invariant manner, so that any transformation of a model will be described in the same way, and the best measure of similarity is obtained at any transformation.

The existing 3D retrieval systems allow the user to perform queries by example. The queried 3D model is then processed, low-level geometrical features are extracted, and similar objects are retrieved from a local database. A shortcoming of the methods that have been proposed so far regarding the $3 \mathrm{D}$ object retrieval, is that neither is the semantic information (high-level features) attached to the (low-level) geometric features of the $3 \mathrm{D}$ content, nor are the personalization options taken into account, which would significantly improve the retrieved results. Moreover, few systems exist so far to take into account annotation and relevance feedback techniques, which are very popular among the corresponding content-based image retrieval systems (CBIR).

Most existing CBIR systems using knowledge either annotate all the objects in the database (full annotation) or annotate a subset of the database manually selected (partial annotation). As the database becomes larger, full annotation is increasingly difficult because of the manual effort needed. Partial annotation is relatively affordable and trims down the heavy manual labor. Once the database is partially annotated, traditional image analysis methods are used to derive semantics of the objects not yet annotated. However, it is not clear "how much" annotation is sufficient for a specific database and what the best subset of objects to annotate is. In other words how the knowledge will be propagated. Such techniques have not been presented so far regarding the $3 \mathrm{D}$ case.

Relevance feedback was first proposed as an interactive tool in text-based retrieval. Since then it has been proven to be a powerful tool and has become a major focus of research in the area of content-based search and retrieval. In the traditional computer centric approaches, which have been proposed so far, the "best" representations and weights are fixed and they cannot effectively model high-level concepts and user's perception subjectivity. In order to overcome these limitations of the computer centric approach, techniques based on relevant feedback, in which the human and computer interact to refine high-level queries to representations based on low-level features, should be developed.

The aim of this special issue is to focus on recent developments in this expanding research area. The special issue will focus on novel approaches in $3 \mathrm{D}$ object retrieval, transforms and methods for efficient geometric feature extraction, annotation and relevance feedback techniques, knowledge propagation (e.g., using Bayesian networks), and their combinations so as to produce a single, powerful, and dominant solution.

Topics of interest include (but are not limited to):

- 3D content-based search and retrieval methods (volume/surface-based)

- Partial matching of 3D objects

- Rotation invariant feature extraction methods for 3D objects 
- Graph-based and topology-based methods

- 3D data and knowledge representation

- Semantic and knowledge propagation over heterogeneous metadata types

- Annotation and relevance feedback techniques for 3D objects

Authors should follow the EURASIP JASP manuscript format described at http://www.hindawi.com/journals/asp/. Prospective authors should submit an electronic copy of their complete manuscript through the EURASIP JASP manuscript tracking system at http://www.mstracking.com/asp/, according to the following timetable:

\begin{tabular}{|l|l|}
\hline Manuscript Due & February 1,2006 \\
\hline Acceptance Notification & June 1,2006 \\
\hline Final Manuscript Due & September 1,2006 \\
\hline Publication Date & 4th Quarter, 2006 \\
\hline
\end{tabular}

\section{GUEST EDITORS:}

Petros Daras, Informatics and Telematics Institute, Centre for Research and Technology Hellas, 57001 Thermi, Thessaloniki, Greece; daras@iti.gr

Ming Ouhyoung, National Taiwan University, Taipei 106, Taiwan; ming@csie.ntu.edu.tw

Tsuhan Chen, Carnegie Mellon University, Pittsburgh, PA 15213, USA; tsuhan@cmu.edu 


\section{Special Issue on}

\section{Robust Speech Recognition}

\section{Call for Papers}

Robustness can be defined as the ability of a system to maintain performance or degrade gracefully when exposed to conditions not well represented in the data used to develop the system. In automatic speech recognition (ASR), systems must be robust to many forms of signal degradation, including speaker characteristics (e.g., dialect and accent), ambient environment (e.g., cellular telephony), transmission channel (e.g., voice over IP), and language (e.g., new words, dialect switching). Robust ASR systems, which have been under development for the past 35 years, have made great progress over the years closing the gap between performance on pristine research tasks and noisy operational data.

However, in recent years, demand is emerging for a new class of systems that tolerate extreme and unpredictable variations in operating conditions. For example, in a cellular telephony environment, there are many nonstationary forms of noise (e.g., multiple speakers) and significant variations in microphone type, position, and placement. Harsh ambient conditions typical in automotive and mobile applications pose similar challenges. Development of systems in a language or dialect for which there is limited or no training data in a target language has become a critical issue for a new generation of voice mining applications. The existence of multiple conditions in a single stream, a situation common to broadcast news applications, and that often involves unpredictable changes in speaker, topic, dialect, or language, is another form of robustness that has gained attention in recent years.

Statistical methods have dominated the field since the early 1980s. Such systems tend to excel at learning the characteristics of large databases that represent good models of the operational conditions and do not generalize well to new environments.

This special issue will focus on recent developments in this key research area. Topics of interest include (but are not limited to):

- Channel and microphone normalization

- Stationary and nonstationary noise modeling, compensation, and/or rejection

- Localization and separation of sound sources (including speaker segregation)
- Signal processing and feature extraction for applications involving hands-free microphones

- Noise robust speech modeling

- Adaptive training techniques

- Rapid adaptation and learning

- Integration of confidence scoring, metadata, and other alternative information sources

- Audio-visual fusion

- Assessment relative to human performance

- Machine learning algorithms for robustness

- Transmission robustness

- Pronunciation modeling

Authors should follow the EURASIP JASP manuscript format described at http://www.hindawi.com/journals/asp/. Prospective authors should submit an electronic copy of their complete manuscript through the EURASIP JASP manuscript tracking system at http://www.mstracking.com/asp/, according to the following timetable:

\begin{tabular}{|l|l|}
\hline Manuscript Due & February 1,2006 \\
\hline Acceptance Notification & June 1, 2006 \\
\hline Final Manuscript Due & September 1,2006 \\
\hline Publication Date & 4th Quarter, 2006 \\
\hline
\end{tabular}

\section{GUEST EDITORS:}

Herve Bourlard, IDIAP Research Institute, Swiss Federal Institute of Technology at Lausanne (EPFL), 1920 Martigny, Switzerland; herve.bourlard@idiap.ch

Mark Gales, Department of Engineering, University of Cambridge, Cambridge CB2 1PZ, UK; mjfg@eng.cam.ac.uk

Maurizio Omologo, ITC-IRST, 38050 Trento, Italy; omologo@itc.it

S. Parthasarathy, AT\&T Labs - Research, NJ 07748, USA; sps@research.att.com

Joe Picone, Department of Electrical and Computer Engineering, Mississippi State University, MS 39762-9571, USA; picone@cavs.msstate.edu 


\section{Special Issue on}

\section{Signal Processing Technologies for Ambient Intelligence in Home-Care Applications}

\section{Call for Papers}

The possibility of allowing elderly people with different kinds of disabilities to conduct a normal life at home and achieve a more effective inclusion in the society is attracting more and more interest from both industrial and governmental bodies (hospitals, healthcare institutions, and social institutions). Ambient intelligence technologies, supported by adequate networks of sensors and actuators, as well as by suitable processing and communication technologies, could enable such an ambitious objective.

Recent researches demonstrated the possibility of providing constant monitoring of environmental and biomedical parameters, and the possibility to autonomously originate alarms, provide primary healthcare services, activate emergency calls, and rescue operations through distributed assistance infrastructures. Nevertheless, several technological challenges are still connected with these applications, ranging from the development of enabling technologies (hardware and software), to the standardization of interfaces, the development of intuitive and ergonomic human-machine interfaces, and the integration of complex systems in a highly multidisciplinary environment.

The objective of this special issue is to collect the most significant contributions and visions coming from both academic and applied research bodies working in this stimulating research field. This is a highly interdisciplinary field comprising many areas, such as signal processing, image processing, computer vision, sensor fusion, machine learning, pattern recognition, biomedical signal processing, multimedia, human-computer interfaces, and networking.

The focus will be primarily on the presentation of original and unpublished works dealing with ambient intelligence and domotic technologies that can enable the provision of advanced homecare services.

This special issue will focus on recent developments in this key research area. Topics of interest include (but are not limited to):

- Video-based monitoring of domestic environments and users

- Continuous versus event-driven monitoring

- Distributed information processing
- Data fusion techniques for event association and automatic alarm generation

- Modeling, detection, and learning of user habits for automatic detection of anomalous behaviors

- Integration of biomedical and behavioral data

- Posture and gait recognition and classification

- Interactive multimedia communications for remote assistance

- Content-based encoding of medical and behavioral data

- Networking support for remote healthcare

- Intelligent/natural man-machine interaction, personalization, and user acceptance

Authors should follow the EURASIP JASP manuscript format described at http://www.hindawi.com/journals/asp/. Prospective authors should submit an electronic copy of their complete manuscripts through the EURASIP JASP manuscript tracking system at http://www.mstracking.com/asp/, according to the following timetable:

\begin{tabular}{|l|l|}
\hline Manuscript Due & March 1, 2006 \\
\hline Acceptance Notification & July 1,2006 \\
\hline Final Manuscript Due & October 1,2006 \\
\hline Publication Date & 1st Quarter, 2007 \\
\hline
\end{tabular}

\section{GUEST EDITORS:}

Francesco G. B. De Natale, Department of Information and Communication Technology, University of Trento, Via Sommarive 14, 38050 Trento, Italy; denatale@ing.unitn.it

Aggelos K. Katsaggelos, Department of Electrical and Computer Engineering, Northwestern University, 2145 Sheridan Road, Evanston, IL 60208-3118, USA; aggk@ece.northwestern.edu

Oscar Mayora, Create-Net Association, Via Solteri 38, 38100 Trento, Italy; oscar.mayora@create-net.it

Ying Wu, Department of Electrical and Computer Engineering, Northwestern University, 2145 Sheridan Road, Evanston, IL 60208-3118, USA; yingwu@ece.northwestern.edu 


\section{Special Issue on}

\section{Spatial Sound and Virtual Acoustics}

\section{Call for Papers}

Spatial sound reproduction has become widespread in the form of multichannel audio, particularly through home theater systems. Reproduction systems from binaural (by headphones) to hundreds of loudspeaker channels (such as wave field synthesis) are entering practical use. The application potential of spatial sound is much wider than multichannel sound, however, and research in the field is active. Spatial sound covers for example the capturing, analysis, coding, synthesis, reproduction, and perception of spatial aspects in audio and acoustics.

In addition to the topics mentioned above, research in virtual acoustics broadens the field. Virtual acoustics includes techniques and methods to create realistic percepts of sound sources and acoustic environments that do not exist naturally but are rendered by advanced reproduction systems using loudspeakers or headphones. Augmented acoustic and audio environments contain both real and virtual acoustic components.

Spatial sound and virtual acoustics are among the major research and application areas in audio signal processing. Topics of active study range from new basic research ideas to improvement of existing applications. Understanding of spatial sound perception by humans is also an important area, in fact a prerequisite to advanced forms of spatial sound and virtual acoustics technology.

This special issue will focus on recent developments in this key research area. Topics of interest include (but are not limited to):

- Multichannel reproduction

- Wave field synthesis

- Binaural reproduction

- Format conversion and enhancement of spatial sound

- Spatial sound recording

- Analysis, synthesis, and coding of spatial sound

- Spatial sound perception and auditory modeling

- Simulation and modeling of room acoustics

- Auralization techniques

- Beamforming and sound source localization

- Acoustic and auditory scene analysis

- Augmented reality audio
- Virtual acoustics (sound environments and sources)

- Intelligent audio environments

- Loudspeaker-room interaction and equalization

- Applications

Authors should follow the EURASIP JASP manuscript format described at http://www.hindawi.com/journals/asp/. Prospective authors should submit an electronic copy of their complete manuscript through the EURASIP JASP manuscript tracking system at http://www.mstracking.com/asp/, according to the following timetable:

\begin{tabular}{|l|l|}
\hline Manuscript Due & May 1,2006 \\
\hline Acceptance Notification & September 1,2006 \\
\hline Final Manuscript Due & December 1,2006 \\
\hline Publication Date & 1st Quarter, 2007 \\
\hline
\end{tabular}

\section{GUEST EDITORS:}

Ville Pulkki, Helsinki University of Technology, Espoo, Finland; ville@acoustics.hut.fi

Christof Faller, EPFL, Lausanne, Switzerland; christof.faller@epfl.ch

Aki Harma, Philips Research Labs, Eindhoven, The Netherlands; aki.harma@philips.com

Tapio Lokki, Helsinki University of Technology, Espoo, Finland; ktlokki@cc.hut.fi

Werner de Bruijn, Philips Research Labs, Eindhoven, The Netherlands; werner.de.bruijn@philips.com 


\section{Special Issue on}

\section{Advances in Electrocardiogram Signal Processing and Analysis}

\section{Call for Papers}

Since its invention in the 19th century when it was little more than a scientific curiosity, the electrocardiogram (ECG) has developed into one of the most important and widely used quantitative diagnostic tools in medicine. It is essential for the identification of disorders of the cardiac rhythm, extremely useful for the diagnosis and management of heart abnormalities such as myocardial infarction (heart attack), and offers helpful clues to the presence of generalised disorders that affect the rest of the body, such as electrolyte disturbances and drug intoxication.

Recording and analysis of the ECG now involves a considerable amount of signal processing for $\mathrm{S} / \mathrm{N}$ enhancement, beat detection, automated classification, and compression. These involve a whole variety of innovative signal processing methods, including adaptive techniques, time-frequency and time-scale procedures, artificial neural networks and fuzzy logic, higher-order statistics and nonlinear schemes, fractals, hierarchical trees, Bayesian approaches, and parametric models, amongst others.

This special issue will review the current status of ECG signal processing and analysis, with particular regard to recent innovations. It will report major achievements of academic and commercial research institutions and individuals, and provide an insight into future developments within this exciting and challenging area.

This special issue will focus on recent developments in this key research area. Topics of interest include (but are not limited to):

- Beat (QRS complex) detection

- ECG compression

- Denoising of ECG signals

- Morphological studies and classification

- ECG modeling techniques

- Expert systems and automated diagnosis

- QT interval measurement and heart-rate variability

- Arrhythmia and ischemia detection and analysis

- Interaction between cardiovascular signals (ECG, blood pressure, respiration, etc.)
- Intracardiac ECG analysis (implantable cardiovascular devices, and pacemakers)

- ECGs and sleep apnoea

- Real-time processing and instrumentation

- ECG telemedicine and e-medicine

- Fetal ECG detection and analysis

- Computational tools and databases for ECG education and research

Authors should follow the EURASIP JASP manuscript format described at http://www.hindawi.com/journals/asp/. Prospective authors should submit an electronic copy of their complete manuscripts through the EURASIP JASP manuscript tracking system at http://www.mstracking.com/asp/, according to the following timetable:

\begin{tabular}{|l|l|}
\hline Manuscript Due & May 1,2006 \\
\hline Acceptance Notification & September 1,2006 \\
\hline Final Manuscript Due & December 1,2006 \\
\hline Publication Date & 1st Quarter, 2007 \\
\hline
\end{tabular}

\section{GUEST EDITORS:}

William Sandham, Scotsig, Glasgow G12 9pf, UK; w.sandham@scotsig.co.uk

David Hamilton, Department of Electronic and Electrical Engineering, University of Strathclyde, Glasgow G1 1XW, UK; d.hamilton@eee.strath.ac.uk

Pablo Laguna Lasaosa, Departmento de Ingeniería Electrónica y Communicaciones, Universidad de Zaragoza, 50015 Zaragoza, Spain; laguna@unizar.es

Maurice Cohen, University of California, San Francisco, USA; mcohen@fresno.ucsf.edu 


\section{Special Issue on}

\section{Emerging Signal Processing Techniques for Power Quality Applications}

\section{Call for Papers}

Recently, end users and utility companies are increasingly concerned with perturbations originated from electrical power quality variations. Investigations are being carried out to completely characterize not only the old traditional type of problems, but also new ones that have arisen as a result of massive use of nonlinear loads and electronics-based equipment in residences, commercial centers, and industrial plants. These nonlinear load effects are aggravated by massive power system interconnections, increasing number of different power sources, and climatic changes.

In order to improve the capability of equipments applied to monitoring the power quality of transmission and distribution power lines, power systems have been facing new analysis and synthesis paradigms, mostly supported by signal processing techniques. The analysis and synthesis of emerging power quality and power system problems led to new research frontiers for the signal processing community, focused on the development and combination of computational intelligence, source coding, pattern recognition, multirate systems, statistical estimation, adaptive signal processing, and other digital processing techniques, implemented in either DSP-based, PC-based, or FPGA-based solutions.

The goal of this proposal is to introduce powerful and efficient real-time or almost-real-time signal processing tools for dealing with the emerging power quality problems. These techniques take into account power-line signals and complementary information, such as climatic changes.

This special issue will focus on recent developments in this key research area. Topics of interest include (but are not limited to):

- Detection of transients

- Classification of multiple events

- Identification of isolated and multiple disturbance sources

- Compression of voltage and current data signals

- Location of disturbance sources

- Prediction of transmission and distribution systems failures

- Demand forecasting
- Parameters estimation for fundamental, harmonics, and interharmonics

Digital signal processing techniques applied to power quality applications are a very attractive and stimulating area of research. Its results will provide, in the near future, new standards for the decentralized and real-time monitoring of transmission and distribution systems, allowing to closely follow and predict power system performance. As a result, the power systems will be more easily planned, expanded, controlled, managed, and supervised.

Authors should follow the EURASIP JASP manuscript format described at http://www.hindawi.com/journals/asp/. Prospective authors should submit an electronic copy of their complete manuscripts through the EURASIP JASP manuscript tracking system at http://www.mstracking.com/asp/, according to the following timetable:

\begin{tabular}{|l|l|}
\hline Manuscript Due & May 1, 2006 \\
\hline Acceptance Notification & September 1, 2006 \\
\hline Final Manuscript Due & December 1,2006 \\
\hline Publication Date & 2nd Quarter, 2007 \\
\hline
\end{tabular}

\section{GUEST EDITORS:}

Moisés Vidal Ribeiro, Department of Electrical Circuit, Federal University of Juiz de Fora, CEP 36036-330, Juiz de Fora, Brazil; mribeiro@ieee.org

Jacques Szczupack, Department of Electrical Engineering, Pontifical Catholic University of Rio de Janeiro, CEP 22453-900, Rio de Janeiro, Brazil; jacques@ele.puc-rio.br

M. Reza Iravani, The Edward S. Rogers SR., Department of Electrical and Computer Engineering, University of Toronto, Toronto, ON, Canada M5S 3G4;

iravani@ecf.utoronto.ca 
Irene Yu-Hua Gu, Department of Signals and Systems,

Chalmers University of Technology, SE-412 96, Gothenburg,

Sweden; irenegu@s2.chalmers.se

Pradipta Kishore Dash, C. V. Raman, College of

Engineering Bhubaneswar, Khurda-752054, Orissa, India;

pkdash_india@yahoo.com

Alexander Mamishev, Department of Electrical

Engineering, University of Washington, WA 98195-2500,

Seattle, USA; mamishev@ee.washington.edu 


\section{Special Issue on}

\section{Super-resolution Enhancement of Digital Video}

\section{Call for Papers}

When designing a system for image acquisition, there is generally a desire for high spatial resolution and a wide fieldof-view. To achieve this, a camera system must typically employ small f-number optics. This produces an image with very high spatial-frequency bandwidth at the focal plane. To avoid aliasing caused by undersampling, the corresponding focal plane array (FPA) must be sufficiently dense. However, cost and fabrication complexities may make this impractical. More fundamentally, smaller detectors capture fewer photons, which can lead to potentially severe noise levels in the acquired imagery. Considering these factors, one may choose to accept a certain level of undersampling or to sacrifice some optical resolution and/or field-of-view.

In image super-resolution (SR), postprocessing is used to obtain images with resolutions that go beyond the conventional limits of the uncompensated imaging system. In some systems, the primary limiting factor is the optical resolution of the image in the focal plane as defined by the cut-off frequency of the optics. We use the term "optical SR" to refer to SR methods that aim to create an image with valid spatial-frequency content that goes beyond the cut-off frequency of the optics. Such techniques typically must rely on extensive a priori information. In other image acquisition systems, the limiting factor may be the density of the FPA, subsequent postprocessing requirements, or transmission bitrate constraints that require data compression. We refer to the process of overcoming the limitations of the FPA in order to obtain the full resolution afforded by the selected optics as "detector SR." Note that some methods may seek to perform both optical and detector SR.

Detector SR algorithms generally process a set of lowresolution aliased frames from a video sequence to produce a high-resolution frame. When subpixel relative motion is present between the objects in the scene and the detector array, a unique set of scene samples are acquired for each frame. This provides the mechanism for effectively increasing the spatial sampling rate of the imaging system without reducing the physical size of the detectors.

With increasing interest in surveillance and the proliferation of digital imaging and video, SR has become a rapidly growing field. Recent advances in SR include innovative algorithms, generalized methods, real-time implementations, and novel applications. The purpose of this special issue is to present leading research and development in the area of super-resolution for digital video. Topics of interest for this special issue include but are not limited to:

- Detector and optical SR algorithms for video

- Real-time or near-real-time SR implementations

- Innovative color SR processing

- Novel SR applications such as improved object detection, recognition, and tracking

- Super-resolution from compressed video

- Subpixel image registration and optical flow

Authors should follow the EURASIP JASP manuscript format described at http://www.hindawi.com/journals/asp/. Prospective authors should submit an electronic copy of their complete manuscript through the EURASIP JASP manuscript tracking system at http://www.mstracking.com/asp/, according to the following timetable:

\begin{tabular}{|l|l|}
\hline Manuscript Due & September 1,2006 \\
\hline Acceptance Notification & February 1, 2007 \\
\hline Final Manuscript Due & April 15, 2007 \\
\hline Publication Date & 3rd Quarter, 2007 \\
\hline
\end{tabular}

\section{GUEST EDITORS:}

Russell C. Hardie, Department of Electrical and Computer Engineering, University of Dayton, 300 College Park, Dayton, OH 45469-0026, USA; rhardie@udayton.edu

Richard R. Schultz, Department of Electrical Engineering, University of North Dakota, Upson II Room 160, P.O. Box 7165, Grand Forks, ND 58202-7165, USA; RichardSchultz@mail.und.nodak.edu

Kenneth E. Barner, Department of Electrical and Computer Engineering, University of Delaware, 140 Evans Hall, Newark, DE 19716-3130, USA; barner@ee.udel.edu 


\section{Special Issue on}

\section{Advanced Signal Processing and Computational Intelligence Techniques for Power Line Communications}

\section{Call for Papers}

In recent years, increased demand for fast Internet access and new multimedia services, the development of new and feasible signal processing techniques associated with faster and low-cost digital signal processors, as well as the deregulation of the telecommunications market have placed major emphasis on the value of investigating hostile media, such as powerline (PL) channels for high-rate data transmissions.

Nowadays, some companies are offering powerline communications (PLC) modems with mean and peak bit-rates around $100 \mathrm{Mbps}$ and $200 \mathrm{Mbps}$, respectively. However, advanced broadband powerline communications (BPLC) modems will surpass this performance. For accomplishing it, some special schemes or solutions for coping with the following issues should be addressed: (i) considerable differences between powerline network topologies; (ii) hostile properties of PL channels, such as attenuation proportional to high frequencies and long distances, high-power impulse noise occurrences, time-varying behavior, and strong inter-symbol interference (ISI) effects; (iv) electromagnetic compatibility with other well-established communication systems working in the same spectrum, (v) climatic conditions in different parts of the world; (vii) reliability and QoS guarantee for video and voice transmissions; and (vi) different demands and needs from developed, developing, and poor countries.

These issues can lead to exciting research frontiers with very promising results if signal processing, digital communication, and computational intelligence techniques are effectively and efficiently combined.

The goal of this special issue is to introduce signal processing, digital communication, and computational intelligence tools either individually or in combined form for advancing reliable and powerful future generations of powerline communication solutions that can be suited with for applications in developed, developing, and poor countries.

Topics of interest include (but are not limited to)

- Multicarrier, spread spectrum, and single carrier techniques

- Channel modeling
- Channel coding and equalization techniques

- Multiuser detection and multiple access techniques

- Synchronization techniques

- Impulse noise cancellation techniques

- FPGA, ASIC, and DSP implementation issues of PLC modems

- Error resilience, error concealment, and Joint sourcechannel design methods for video transmission through PL channels

Authors should follow the EURASIP JASP manuscript format described at the journal site http://asp.hindawi.com/. Prospective authors should submit an electronic copy of their complete manuscripts through the EURASIP JASP manuscript tracking system at http://www.mstracking.com/asp/, according to the following timetable:

\begin{tabular}{|l|l|}
\hline Manuscript Due & October 1,2006 \\
\hline Acceptance Notification & January 1, 2007 \\
\hline Final Manuscript Due & April 1, 2007 \\
\hline Publication Date & 3rd Quarter, 2007 \\
\hline
\end{tabular}

\section{GUEST EDITORS:}

Moisés Vidal Ribeiro, Federal University of Juiz de Fora, Brazil; mribeiro@ieee.org

Lutz Lampe, University of British Columbia, Canada; lampe@ece.ubc.ca

Sanjit K. Mitra, University of California, Santa Barbara, USA; mitra@ece.ucsb.edu

Klaus Dostert, University of Karlsruhe, Germany; klaus.dostert@etec.uni-karlsruhe.de

Halid Hrasnica, Dresden University of Technology, Germanyhrasnica@ifn.et.tu-dresden.de 


\section{Special Issue on}

\section{Video Adaptation for Heterogeneous Environments}

\section{Call for Papers}

The explosive growth of compressed video streams and repositories accessible worldwide, the recent addition of new video-related standards such as H.264/AVC, MPEG-7, and MPEG-21, and the ever-increasing prevalence of heterogeneous, video-enabled terminals such as computer, TV, mobile phones, and personal digital assistants have escalated the need for efficient and effective techniques for adapting compressed videos to better suit the different capabilities, constraints, and requirements of various transmission networks, applications, and end users. For instance, Universal Multimedia Access (UMA) advocates the provision and adaptation of the same multimedia content for different networks, terminals, and user preferences.

Video adaptation is an emerging field that offers a rich body of knowledge and techniques for handling the huge variation of resource constraints (e.g., bandwidth, display capability, processing speed, and power consumption) and the large diversity of user tasks in pervasive media applications. Considerable amounts of research and development activities in industry and academia have been devoted to answering the many challenges in making better use of video content across systems and applications of various kinds.

Video adaptation may apply to individual or multiple video streams and may call for different means depending on the objectives and requirements of adaptation. Transcoding, transmoding (cross-modality transcoding), scalable content representation, content abstraction and summarization are popular means for video adaptation. In addition, video content analysis and understanding, including low-level feature analysis and high-level semantics understanding, play an important role in video adaptation as essential video content can be better preserved.

The aim of this special issue is to present state-of-theart developments in this flourishing and important research field. Contributions in theoretical study, architecture design, performance analysis, complexity reduction, and real-world applications are all welcome.

Topics of interest include (but are not limited to):

- Heterogeneous video transcoding

- Scalable video coding

- Dynamic bitstream switching for video adaptation
- Signal, structural, and semantic-level video adaptation

- Content analysis and understanding for video adaptation

- Video summarization and abstraction

- Copyright protection for video adaptation

- Crossmedia techniques for video adaptation

- Testing, field trials, and applications of video adaptation services

- International standard activities for video adaptation

Authors should follow the EURASIP JASP manuscript format described at http://www.hindawi.com/journals/asp/. Prospective authors should submit an electronic copy of their complete manuscript through the EURASIP JASP manuscript tracking system at http://www.mstracking.com/asp/, according to the following timetable:

\begin{tabular}{|l|l|}
\hline Manuscript Due & September 1,2006 \\
\hline Acceptance Notification & January 1,2007 \\
\hline Final Manuscript Due & April 1, 2007 \\
\hline Publication Date & 3rd Quarter 2007 \\
\hline
\end{tabular}

\section{GUEST EDITORS:}

Chia-Wen Lin, Department of Computer Science and Information Engineering, National Chung Cheng University, Chiayi 621, Taiwan; cwlin@cs.ccu.edu.tw

Yap-Peng Tan, School of Electrical and Electronic Engineering, Nanyang Technological University, Nanyang Avenue, Singapore 639798, Singapore; eyptan@ntu.edu.sg

Ming-Ting Sun, Department of Electrical Engineering, University of Washington, Seattle, WA 98195, USA ; sun@ee.washington.edu

Alex Kot, School of Electrical and Electronic Engineering, Nanyang Technological University, Nanyang Avenue, Singapore 639798, Singapore; eackot@ntu.edu.sg 
Anthony Vetro, Mitsubishi Electric Research Laboratories, 201 Broadway, 8th Floor, Cambridge, MA 02138, USA;

avetro@merl.com 


\section{Special Issue on}

\section{Transforming Signal Processing Applications into Parallel Implementations}

\section{Call for Papers}

There is an increasing need to develop efficient "systemlevel" models, methods, and tools to support designers to quickly transform signal processing application specification to heterogeneous hardware and software architectures such as arrays of DSPs, heterogeneous platforms involving microprocessors, DSPs and FPGAs, and other evolving multiprocessor SoC architectures. Typically, the design process involves aspects of application and architecture modeling as well as transformations to translate the application models to architecture models for subsequent performance analysis and design space exploration. Accurate predictions are indispensable because next generation signal processing applications, for example, audio, video, and array signal processing impose high throughput, real-time and energy constraints that can no longer be served by a single DSP.

There are a number of key issues in transforming application models into parallel implementations that are not addressed in current approaches. These are engineering the application specification, transforming application specification, or representation of the architecture specification as well as communication models such as data transfer and synchronization primitives in both models.

The purpose of this call for papers is to address approaches that include application transformations in the performance, analysis, and design space exploration efforts when taking signal processing applications to concurrent and parallel implementations. The Guest Editors are soliciting contributions in joint application and architecture space exploration that outperform the current architecture-only design space exploration methods and tools.

Topics of interest for this special issue include but are not limited to:

- modeling applications in terms of (abstract) control-dataflow graph, dataflow graph, and process network models of computation (MoC)

- transforming application models or algorithmic engineering

- transforming application MoCs to architecture MoCs

- joint application and architecture space exploration
- joint application and architecture performance analysis

- extending the concept of algorithmic engineering to architecture engineering

- design cases and applications mapped on multiprocessor, homogeneous, or heterogeneous SOCs, showing joint optimization of application and architecture

Authors should follow the EURASIP JASP manuscript format described at http://www.hindawi.com/journals/asp/. Prospective authors should submit an electronic copy of their complete manuscript through the EURASIP JASP manuscript tracking system at http://www.mstracking.com/asp/, according to the following timetable:

\begin{tabular}{|l|l|}
\hline Manuscript Due & September 1,2006 \\
\hline Acceptance Notification & January 1, 2007 \\
\hline Final Manuscript Due & April 1, 2007 \\
\hline Publication Date & 3rd Quarter 2007 \\
\hline
\end{tabular}

\section{GUEST EDITORS:}

F. Deprettre, Leiden Embedded Research Center, Leiden University, Niels Bohrweg 1, 2333 CA Leiden, The Netherlands; edd@liacs.nl

Roger Woods, School of Electrical and Electronic Engineering, Queens University of Belfast, Ashby Building, Stranmillis Road, Belfast, BT9 5AH, UK; r.woods@qub.ac.uk

Ingrid Verbauwhede, Katholieke Universiteit Leuven, ESAT-COSIC, Kasteelpark Arenberg 10, 3001 Leuven, Belgium; Ingrid.verbauwhede@esat.kuleuven.be

Erwin de Kock, Philips Research, High Tech Campus 31, 5656 AE Eindhoven, The Netherlands; erwin.de.kock@philips.com 

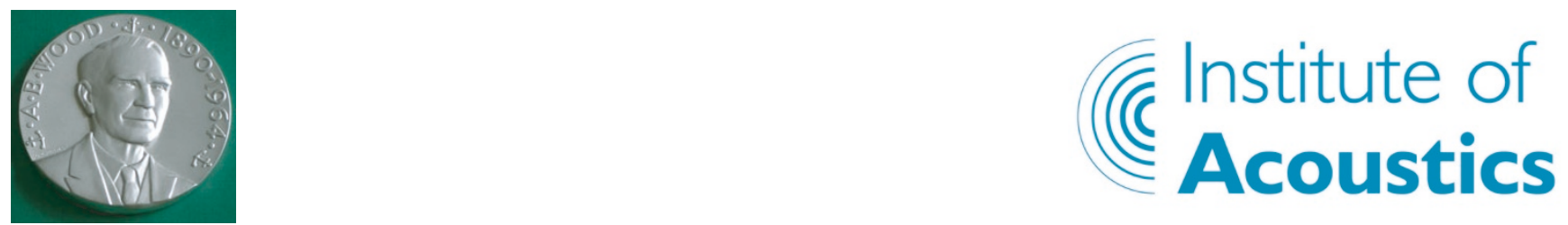

NEWS RELEASE

Nominations Invited for the Institute of Acoustics 2006 A B Wood Medal

The Institute of Acoustics, the UK's leading professional body for those working in acoustics, noise and vibration, is inviting nominations for its prestigious A B Wood Medal for the year 2006.

The A B Wood Medal and prize is presented to an individual, usually under the age of 35 , for distinguished contributions to the application of underwater acoustics. The award is made annually, in even numbered years to a person from Europe and in odd numbered years to someone from the USA/Canada. The 2005 Medal was awarded to Dr A Thode from the USA for his innovative, interdisciplinary research in ocean and marine mammal acoustics.

Nominations should consist of the candidate's CV, clearly identifying peer reviewed publications, and a letter of endorsement from the nominator identifying the contribution the candidate has made to underwater acoustics. In addition, there should be a further reference from a person involved in underwater acoustics and not closely associated with the candidate. Nominees should be citizens of a European Union country for the 2006 Medal. Nominations should be marked confidential and addressed to the President of the Institute of Acoustics at 77A St Peter's Street, St. Albans, Herts, AL1 3BN. The deadline for receipt of nominations is $\mathbf{1 5}$ October 2005.

Dr Tony Jones, President of the Institute of Acoustics, comments, "A B Wood was a modest man who took delight in helping his younger colleagues. It is therefore appropriate that this prestigious award should be designed to recognise the contributions of young acousticians."

Further information and an nomination form can be found on the Institute's website at www.ioa.org.uk.

\footnotetext{
A B Wood

Albert Beaumont Wood was born in Yorkshire in 1890 and graduated from Manchester University in 1912. He became one of the first two research scientists at the Admiralty to
}

work on antisubmarine defence. He designed the first directional hydrophone and was well known for the many contributions he made to the science of underwater acoustics and for the help he gave to younger colleagues. The medal was instituted after his death by his many friends on both sides of the Atlantic and was administered by the Institute of Physics until the formation of the Institute of Acoustics in 1974.

\section{PRESS CONTACT}

\section{Judy Edrich}

Publicity \& Information Manager, Institute of Acoustics Tel: 01727 848195; E-mail: judy.edrich@ioa.org.uk

\section{EDITORS NOTES}

The Institute of Acoustics is the UK's professional body for those working in acoustics, noise and vibration. It was formed in 1974 from the amalgamation of the Acoustics Group of the Institute of Physics and the British Acoustical Society (a daughter society of the Institution of Mechanical Engineers). The Institute of Acoustics is a nominated body of the Engineering Council, offering registration at Chartered and Incorporated Engineer levels.

The Institute has some 2500 members from a rich diversity of backgrounds, with engineers, scientists, educators, lawyers, occupational hygienists, architects and environmental health officers among their number. This multidisciplinary culture provides a productive environment for cross-fertilisation of ideas and initiatives. The range of interests of members within the world of acoustics is equally wide, embracing such aspects as aerodynamics, architectural acoustics, building acoustics, electroacoustics, engineering dynamics, noise and vibration, hearing, speech, underwater acoustics, together with a variety of environmental aspects. The lively nature of the Institute is demonstrated by the breadth of its learned society programmes.

For more information please visit our site at www.ioa.org.uk. 


\title{
MULTIMEDIA FINGERPRINTING FORENSICS FOR TRAITOR TRACING
}

\author{
Edited by: K. J. Ray Liu, Wade Trappe, Z. Jane Wang, Min Wu, and Hong Zhao
}

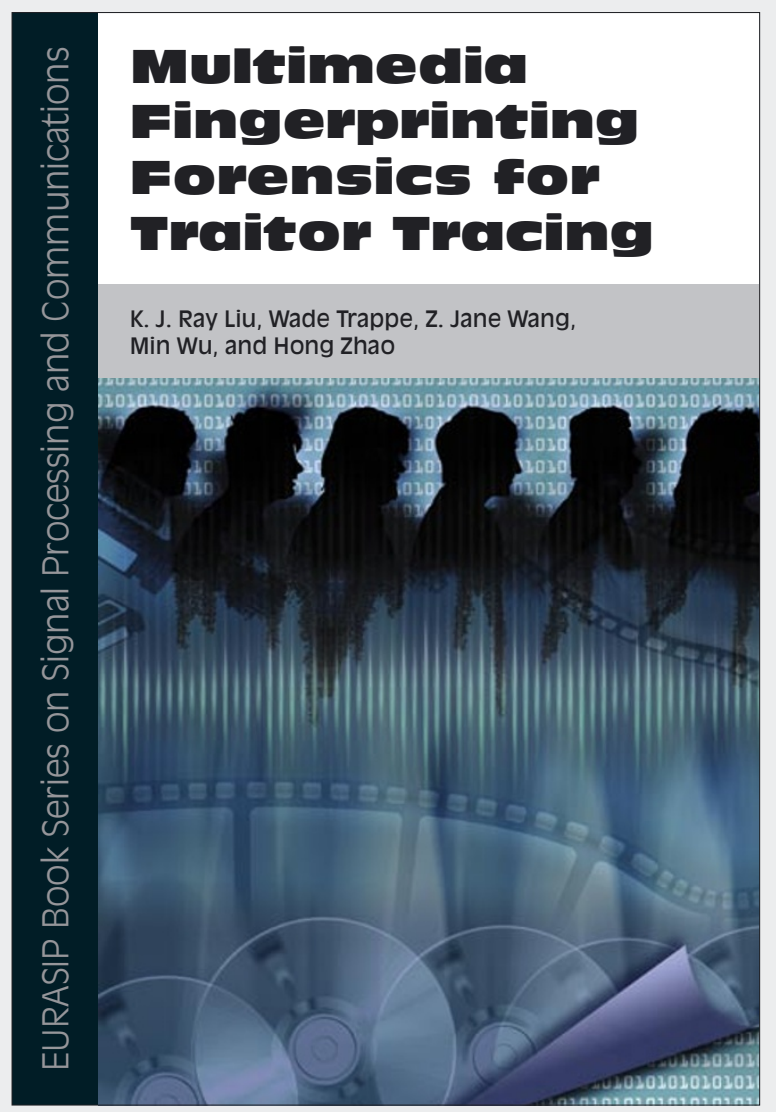

The popularity of multimedia content has led to the widespread distribution and consumption of digital multimedia data. As a result of the relative ease with which individuals may now alter and repackage digital content, ensuring that media content is employed by authorized users for its intended purpose is becoming an issue of eminent importance to both governmental security and commercial applications. Digital fingerprinting is a class of multimedia forensic technologies to track and identify entities involved in the illegal manipulation and unauthorized usage of multimedia content, thereby protecting the sensitive nature of multimedia data as well as its commercial value after the content has been delivered to a recipient.

"Multimedia Fingerprinting Forensics for Traitor Tracing" covers the essential aspects of research in this emerging technology, and explains the latest development in this field. It describes the framework of multimedia fingerprinting, discusses the challenges that may be faced when enforcing usage policies, and investigates the design of fingerprints that cope with new families of multiuser attacks that may be mounted against media fingerprints. The discussion provided in the book highlights challenging problems as well as future trends in this research field, providing readers with a broader view of the evolution of the young field of multimedia forensics.

Topics and features:

Comprehensive coverage of digital watermarking and fingerprinting in multimedia forensics for a number of media types; Detailed discussion on challenges in multimedia fingerprinting and analysis of effective multiuser collusion attacks on digital fingerprinting; Thorough investigation of fingerprint design and performance analysis for addressing different application concerns arising in multimedia fingerprinting; Well-organized explanation of problems and solutions, such as order-statistics-based nonlinear collusion attacks, efficient detection and identification of colluders, group-oriented fingerprint design, and anticollusion codes for multimedia fingerprinting.

For more information and online orders, please visit http://www.hindawi.com/books/spc/volume-4/ For any inquires on how to order this title, please contact books.orders@hindawi.com

The EURASIP Book Series on Signal Processing and Communications publishes monographs, edited volumes, and textbooks on Signal Processing and Communications. For more information about the series, please visit http://hindawi.com/books/spc/about.html 


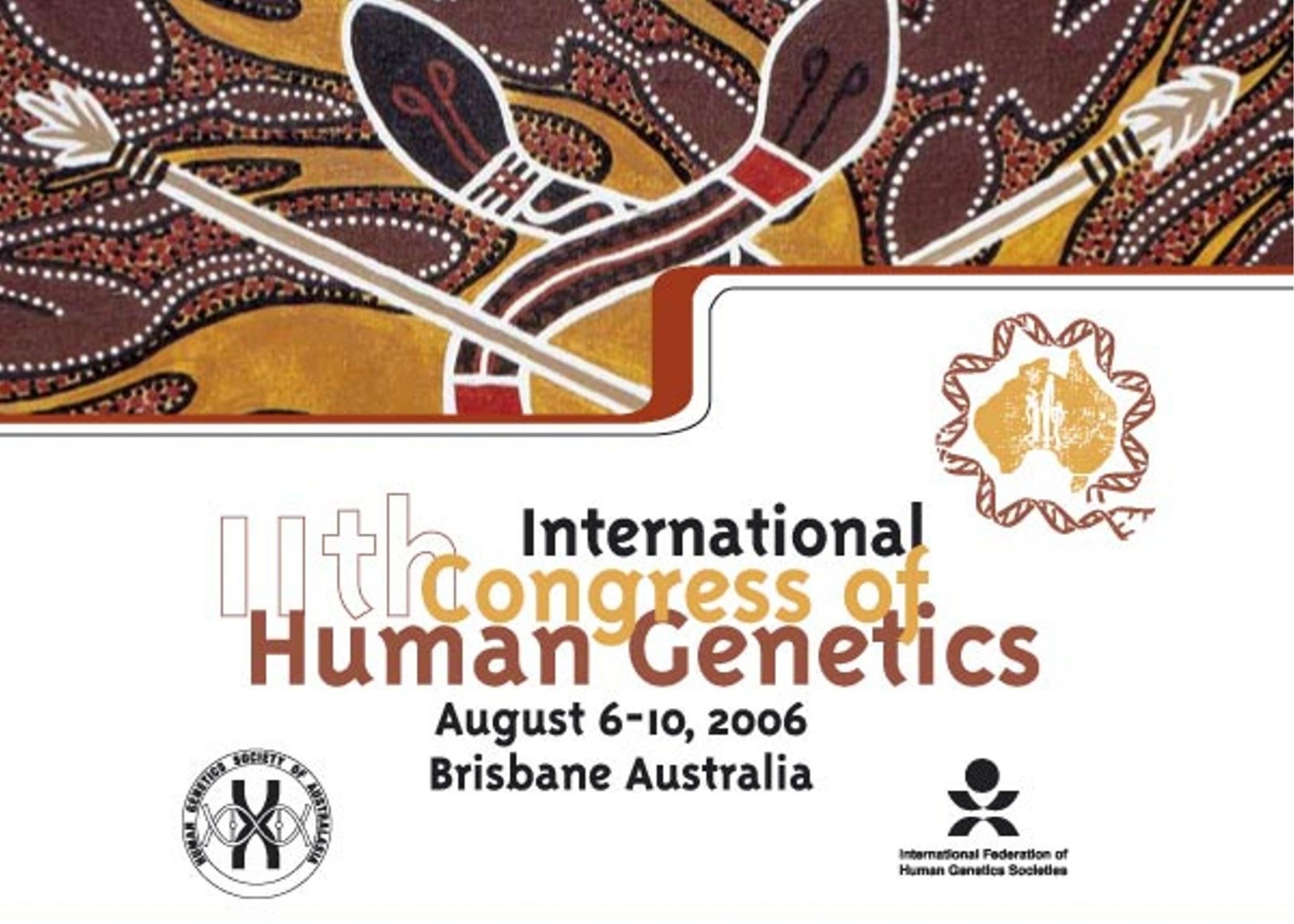

Register now @ www.ichg2006.com

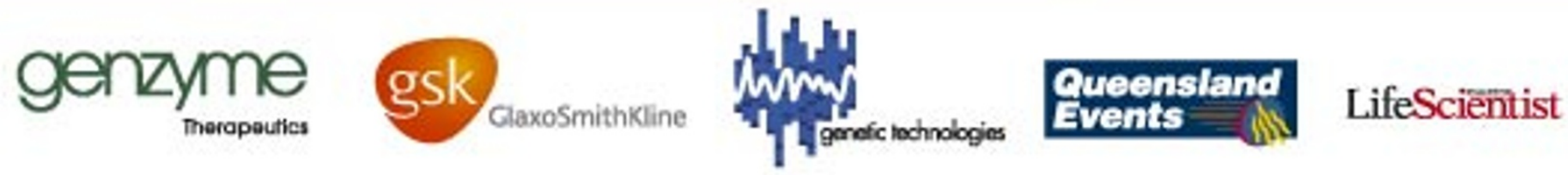

Congress Secretariat:

ICHG 2006 Congress Secretariat

CI-ICMS Pty Ltd,

PO BOX 3496, South Brisbane Queensland 4101, Australia Phone: $+61738441138 \mathrm{Fax}:+61738440909$

Email: ichg2006\$icms. com.au

\section{www.ichg2006.com}

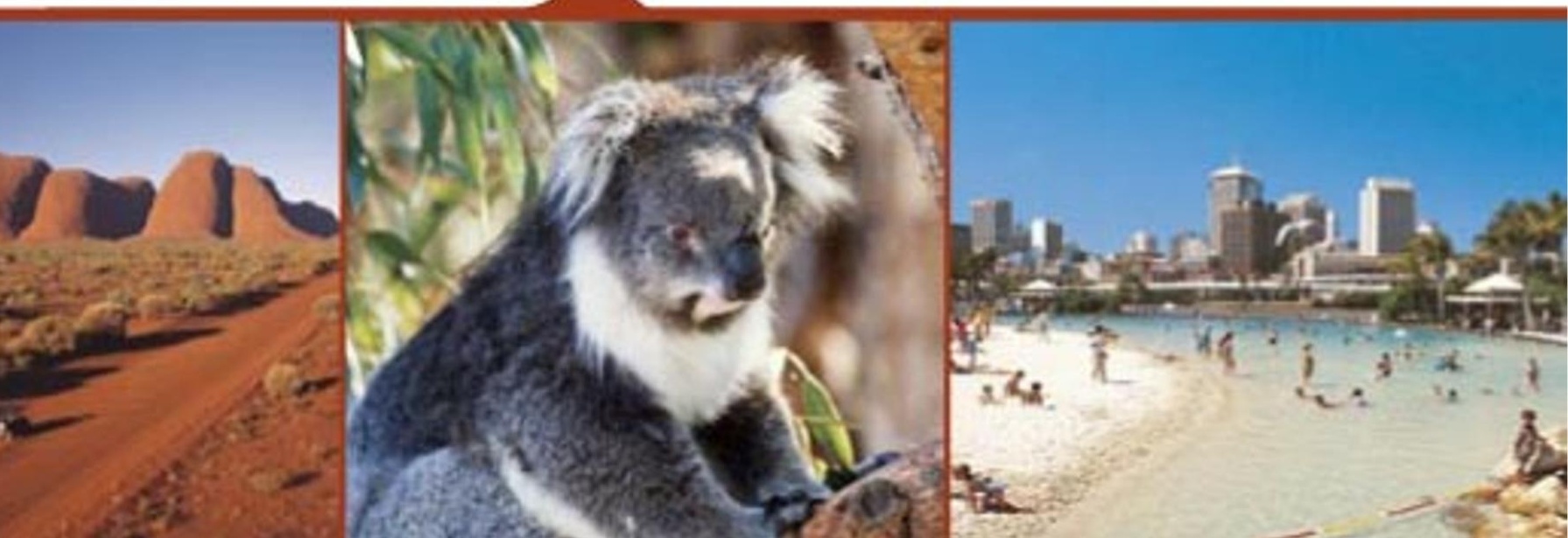




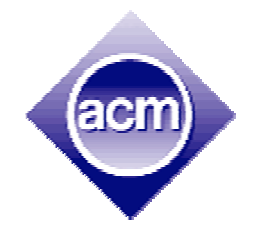

ACM Multimedia and Security

Workshop

September 26-27, 2006

Organizing Committee

General Chair

Sviatoslav Voloshynovskiy

University of Geneva, Switzerland

Local Arrangement Chair and Treasurer

Oleksiy Koval

University of Geneva, Switzerland

\section{Program Chairs}

Jana Dittmann

Otto-von-Guericke University Magdeburg,

Germany

Jessica Fridrich

SUNY Binghamton, USA

\section{ACM SIGMM Chair \\ Ramesh Jain \\ Georgia Tech, USA}

\section{Program Committee}

Mauro Barni

Ahmet M. Eskicioglu

Teddy Furon

Mariam Haroutunian

Ton Kalker

Stefan Katzenbeisser

Deepa Kundur

Inald Lagendijk

Heung-Kyo Lee

Nasir Memon

M. Kivanc Mihcak

Pierre Moulin

Fernando Perez-Gonzalez

Thierry Pun

Claus Vielhauer

Min Wu

\section{Time Schedule}

Submissions start: February 1

Submissions end: April 20

Authors notified: July 20

Camera ready by: August 10

Workshop: September 26-27, 2006

\section{CALL FOR PAPERS}

$8^{\text {th }}$ ACM Multimedia Security Workshop

September 26-27, 2006

The objective of this 8th ACM Multimedia and Security Workshop is to identify the key issues to be addressed by future research in the areas of media manipulation recognition, media data authentication, and detection of hidden communication channels. We expect the workshop to help motivate this research and to establish fruitful relationships with the key actors from academia, industry, and government in the US and European and Asian countries. It will consist of invited papers, full papers, short papers, and possibly a rump or a panel session. This event continues a successful series of workshops started in 1998.

\section{OBJECTIVES}

- Discussion of emerging technologies in digital multi-media authentication, identification, fingerprinting, and steganalysis

- Identification of key research problems with the biggest impact on specified deficiencies in the field of secure multimedia distribution

- Formulation of target applications of identified technologies (in both the commercial and military sectors)

- Discussion of legal issues connected to multimedia security, digital watermarking, and steganography

\section{SCOPE AND PAPERS}

Papers should address theoretical and practical issues of multimedia watermarking and steganography. We will consider both theoretical papers dealing with fundamental issues and application oriented contributions (software and hardware demos are highly encouraged). Topics include but are not limited to:

- Robust watermarking of multimedia

- Authentication of multimedia

- Informational-theoretical aspects of data hiding

- Steganography and steganalysis

- Forensic analysis of digital multimedia

- Practical systems with aspects of data hiding

- Watermarking quality evaluation and benchmarks

- New applications, security issues, and legal aspects

- Data hiding applications in biometrics: document security and person authentication

In particular, the call for papers includes request for full papers with high degree of innovations as well as short papers with interesting improvements of existing art or new ideas and research directions. Full papers should be 6-12 pages long, short papers 4-6 pages long (ACM format http://www.acm.org/sigmm/). Accepted papers will be published in ACM workshop proceedings.

\section{Submission Deadline April 20, 2006}

Authors are invited to submit, by email, full papers or short papers by indicating the type of the paper (full/short) in electronic format (PDF or PostScript) to https://msrcmt.research.microsoft.com/ACM2006. Create a new user account, login, and follow the submission instructions. 


\section{Addaptive Mallitimedia Retrievall}
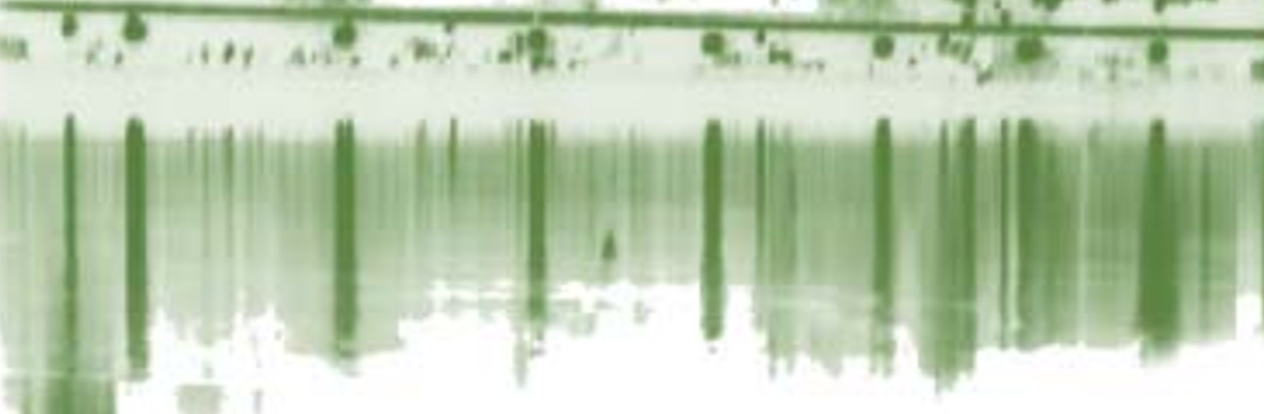

$4^{\text {th }}$ International Workshop on Adaptive Multimedia Retrieval July 27-28, 2006 - University of Geneva, Switzerland

\section{http://viper.unige.ch/amr2006}

The workshop focuses especially on researchers that are working on feature extraction techniques for multimedia, computer linguistic approaches, (dynamic) data analysis methods, and visualization methods as well as user interface design. Therefore, contributions to the workshop should focus on, but are not limited to:

$>$ Multimedia retrieval systems (for text, image, audio, video and mixed-media)

$>$ Theoretical foundations of multimedia retrieval and mining

$>$ Intelligent multimedia data modelling, indexing and structure extraction

$>$ Adaptive Hypermedia and web based systems

$>$ Metadata for multimedia retrieval

$>$ Multimedia and multi-modal mining

> Semantic content analysis for multimedia

$>$ Semantic web and ontologies

$>$ Adaptive query languages

> Similarity measures (especially user adaptive measures)

> User and preference modelling (including feedback models)

$>$ Methods for adaptive data visualization and user interfaces

\section{General Chair:}

$>$ Stéphane Marchand-Maillet University of Geneva

\section{Program Chairs:}

$>$ Eric Bruno

University of Geneva

$>$ Andreas Nürnberger University of Magdeburg

$>$ Marcin Detyniecki LIP6, CNRS Paris

\section{Local Chair:}

$>$ Nicolas Moënne-Loccoz University of Geneva
Deadline for paper submission:

Notification of acceptance/rejection:

Deadline for final paper submission:

Workshop starts:

Submissions should be formatted according to Springer LNCS style. Papers should have about 10 pages but must not exceed 15 pages.

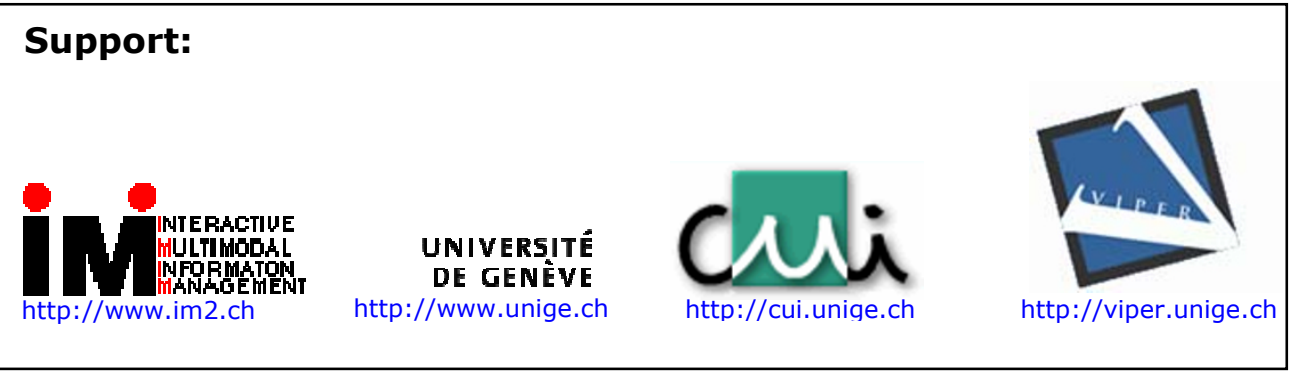


EURASIP Book Series on SP\&C, Volume 2, ISBN 977-5945-07-0

\section{GENOMIC SIGNAL PROCESSING AND STATISTICS}

Edited by: Edward R. Dougherty, llya Shmulevich, Jie Chen, and Z. Jane Wang

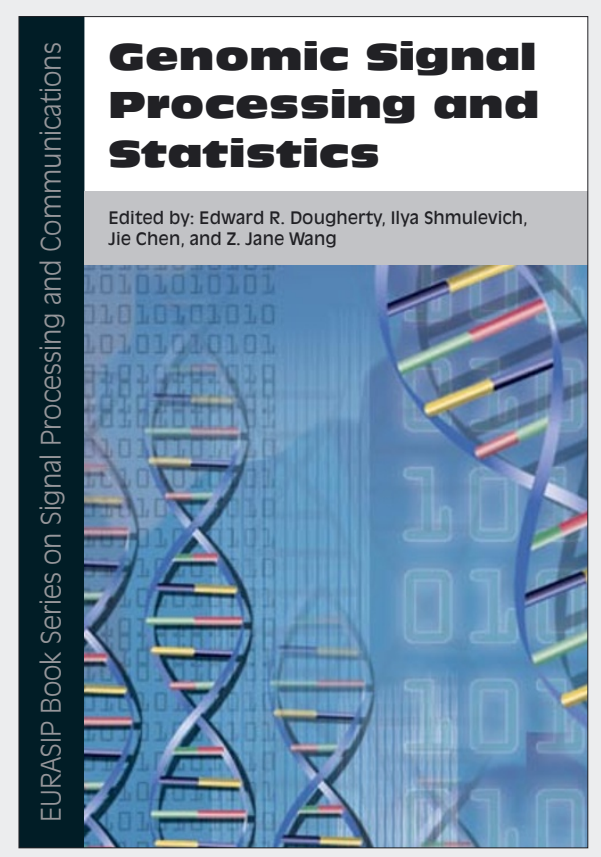

$\mathrm{R}$ ecent advances in genomic studies have stimulated synergetic research and development in many cross-disciplinary areas. Genomic data, especially the recent large-scale microarray gene expression data, represents enormous challenges for signal processing and statistics in processing these vast data to reveal the complex biological functionality. This perspective naturally leads to a new field, genomic signal processing (GSP), which studies the processing of genomic signals by integrating the theory of signal processing and statistics. Written by an international, interdisciplinary team of authors, this invaluable edited volume is accessible to students just entering this emergent field, and to researchers, both in academia and industry, in the fields of molecular biology, engineering, statistics, and signal processing. The book provides tutorial-level overviews and addresses the specific needs of genomic signal processing students and researchers as a reference book.

The book aims to address current genomic challenges by exploiting potential synergies between genomics, signal processing, and statistics, with special emphasis on signal processing and statistical tools for structural and functional understanding of genomic data. The book is partitioned into three parts. In part I, a brief history of genomic research and a background introduction from both biological and signal-processing/ statistical perspectives are provided so that readers can easily follow the material presented in the rest of the book. In part II, overviews of state-of-the-art techniques are provided. We start with a chapter on sequence analysis, and follow with chapters on feature selection, clustering, and classification of microarray data. The next three chapters discuss the modeling, analysis, and simulation of biological regulatory networks, especially gene regulatory networks based on Boolean and Bayesian approaches. The next two chapters treat visualization and compression of gene data, and supercomputer implementation of genomic signal processing systems. Part II concludes with two chapters on systems biology and medical implications of genomic research. Finally, part III discusses the future trends in genomic signal processing and statistics research.

For more information and online orders please visit: http://www.hindawi.com/books/spc/volume-2/ For any inquiries on how to order this title please contact books.orders@hindawi.com

The EURASIP Book Series on Signal Processing and Communications publishes monographs, edited volumes, and textbooks on Signal Processing and Communications. For more information about the series please visit: http://hindawi.com/books/spc/about.html 